\title{
Fetal Spinal Cord Transplants Support Growth of Supraspinal and Segmental Projections after Cervical Spinal Cord Hemisection in the Neonatal Rat
}

\author{
Pamela S. Diener and Barbara S. Bregman \\ Department of Cell Biology, Division of Neurobiology, Georgetown University Medical Center, Washington, D.C. 20007
}

Cervical spinal cord injury at birth permanently disrupts forelimb function in goal-directed reaching. Transplants of fetal spinal cord tissue permit the development of skilled forelimb use and associated postural adjustments (Diener and Bregman, 1998, companion article). The aim of this study was to determine whether transplants of fetal spinal cord tissue support the remodeling of supraspinal and segmental pathways that may underlie recovery of postural reflexes and forelimb movements. Although brainstem-spinal and segmental projections to the cervical spinal cord are present at birth, skilled forelimb reaching has not yet developed. Three-day-old rats received a cervical spinal cord overhemisection with or without transplantation of fetal spinal cord tissue (embryonic day 14); unoperated pups served as normal controls. Neuroanatomical tracing techniques were used to examine the organization of CNS pathways that may influence target-directed reaching. In animals with hemisections only, corticospinal, brainstem-spinal, and dorsal root projections within the spinal cord were decreased in number and extent. In contrast, animals receiving hemisections plus transplants exhibited growth of these projections throughout the transplant and over long distances within the host spinal cord caudal to the transplant. Raphespinal axons were apposed to numerous propriospinal neurons in control and transplant animals; these associations were greatly reduced in the lesion-only animals. These observations suggest that after neonatal cervical spinal cord injury, embryonic transplants support axonal growth of CNS pathways and specifically supraspinal input to propriospinal neurons. We suggest that after neonatal spinal injury in the rat, the transplant-mediated reestablishment of supraspinal input to spinal circuitry is the mechanism underlying the development of target-directed reaching and associated postural adjustments.

Key words: raphespinal axons; rubrospinal axons; corticospinal axons; dorsal root axons; red nucleus; cortex; neuroanatomical tracing
Voluntary control of locomotion requires modulation of the intrinsic activity of central pattern generators by supraspinal and segmental projections (Grillner, 1973, 1975; Andersson et al., 1978). After spinal cord injury in the neonate, transplants of embryonic tissue enhance the recovery of hindlimb locomotion by supporting the regrowth of corticospinal, brainstem-spinal, and segmental axons to hindlimb pattern generators (Kunkel-Bagden and Bregman, 1990; Kunkel-Bagden et al., 1992; Bregman et al., 1993; for review, see Bregman, 1994). Previous studies indicate that axons penetrate the transplant after early thoracic spinal cord injury (Bregman, 1987a,b; Reier et al., 1986; Howland et al., 1995) and elongate for long distances within the host cord caudal to the transplant (Bregman, 1987a,b; Bregman et al., 1989; Bregman and Bernstein-Goral, 1991; Bernstein-Goral and Bregman, 1993). This growth has been correlated with improved performance in rhythmical alternating movements such as locomotion and other developmental reflexes (Bregman and Goldberger, 1983; Breg-

\footnotetext{
Received June 26, 1997; revised Oct. 22, 1997; accepted Oct. 27, 1997.

This work was supported by National Institutes of Health Grants NS27054 and NS19259 from the National Institute of Neurological Disorders and Stroke and Grant T32 HD07459 from the National Center for Medical Rehabilitation Research to B.S.B. We are grateful to Marietta McAtee and Hai Ning Dai for surgical assistance and technical support. We thank Marietta McAtee and Drs. Holli Bernstein-Goral and Melinda Kelley for their helpful comments on this manuscript.

Correspondence should be addressed to Dr. Barbara S. Bregman, Department of Cell Biology, Georgetown University Medical Center, 3900 Reservoir Road NW, Washington, D.C. 20007.

Dr. Diener's present address: Department of Physical Therapy, Marymount University, 2807 N. Glebe Road, Arlington, VA 22207-4299.

Copyright (C) 1998 Society for Neuroscience $\quad 0270-6474 / 98 / 180779-15 \$ 05.00 / 0$
}

man et al., 1993; Howland et al., 1995). After spinal cord lesions and transplants in the adult, host axons regenerate into the transplants, but long-distance axonal growth caudal to the transplant does not occur (Kunkel-Bagden et al., 1992; Bregman, 1994; Bregman et al., 1997). Although essential to locomotor development, the central pattern generator located in forelimb and hindlimb segments of the spinal cord does not form the foundation for skilled forelimb movements. Instead, the propriospinal neurons in the cervical cord integrate information from numerous supraspinal pathways (Alstermark et al., 1981a,b, 1984a,b; Alstermark and Sasaki, 1985) and in turn extend to axons that project directly and indirectly to forelimb and postural musculature (A1stermark et al., 1981a,b, 1984b; Alstermark and Sasaki, 1985). Supraspinal and segmental projections also directly influence brachial and lumbar spinal neurons that contribute to the production of reaching and associated postural adjustments (Illert et al., 1976a,b; Illert and Tanaka, 1978; Alstermark et al., 1984a, 1987b,c,d, 1990, 1991a,b; Alstermark and Sasaki, 1985). Specific reaching and grasping deficits become apparent if this network or the projections to it are disrupted by cervical cord injury in adult rats (Schrimsher and Reier, 1992, 1993). Skilled reaching is not present at birth and fails to develop after neonatal cervical spinal cord injury (Diener and Bregman, 1998, companion article). In contrast, after early high cervical injury and transplants of fetal spinal cord tissue, rats develop complex forelimb tasks such as reaching and accompanying postural adjustments (Diener and Bregman, 1998). Relatively little is known about the capacity for growth of immature supraspinal and propriospinal neurons after 
high cervical spinal cord injury in the neonatal rat. Therefore, our aims were (1) to examine the growth of axotomized supraspinal and segmental pathways into the transplant and into the host cord caudal to a high cervical cord injury and (2) to characterize the regions in which injured axons terminate to suggest potential mechanisms underlying the development of goal-directed forelimb activity. We hypothesized that skilled forelimb movements are acquired because of transplant-supported growth of supraspinal and segmental axons into forelimb and axial regions of the spinal cord, and that such growth would not be observed in lesion-only animals. The results indicate that in the presence of a fetal spinal cord transplant at the neonatal cervical spinal cord lesion site, axotomized supraspinal neurons grow into the transplant and reach normal targets within the host spinal cord caudal to the lesion.

Preliminary results of this work have been published previously in abstract form (Diener and Bregman, 1995).

\section{MATERIALS AND METHODS}

\section{Subjects and spinal cord lesions and transplants}

Newborn Sprague Dawley rat pups [including all rats undergoing behavioral testing (Diener and Bregman, 1998)] were randomly assigned to either operated [overhemisection only ( $\mathrm{HX}), n=25$; overhemisection plus fetal spinal cord transplant (HX $+\mathrm{TP}), n=27]$ or unoperated groups [control group $(\mathrm{CON}), n=29$ ]. Table 1 lists the distribution of animals meeting all criteria for inclusion in the study (see below) by experimental group and neuroanatomical tracing procedure. Surgical techniques were similar to those described previously (Bregman and Reier, 1986; Bregman and McAtee, 1993) and were identical to those used in the companion study (Diener and Bregman, 1998). Briefly, newborn pups $(<72 \mathrm{hr})$ were anesthetized by hypothermia. Under a dissecting microscope, the skin and muscles overlying the cervical cord were separated and retracted. The $\mathrm{C} 2$ and $\mathrm{C} 3$ vertebrae were removed by laminectomy, and the underlying spinal cord segment was exposed by slitting the dural sheath. Iridectomy scissors were used to sever the right side of the cord and the dorsal funiculus, bilaterally. The operated animals were divided into two groups at this point. Half of the animals were placed into the lesion-only group (HX), and the remaining group received transplants of embryonic tissue $(\mathrm{HX}+\mathrm{TP})$.

Preparation of neural tissue transplants has been described in detail elsewhere (Bregman and McAtee, 1993). Briefly, timed-pregnant Sprague Dawley rats (Zivic Miller Laboratories, Zelienople, PA) were anesthetized with chloral hydrate $(400 \mathrm{mg} / \mathrm{kg}$, i.p.) at embryonic day (E) 14. Embryos were removed by Cesarean section every $45 \mathrm{~min}$ as needed and dissected in DMEM. Meninges were stripped from the spinal cord, and cervical segments were isolated and cut into $1-2 \mathrm{~mm}$ pieces. Transplants were inserted into the gap created by the retraction of the cut ends of the spinal cord. It is important to note that at E14, serotonergic projections to the cord have not yet developed (Bregman, 1987a; Bregman and McAtee, 1993). The fetal tissue dissection is done to ensure that no contaminating raphe neurons are contained in the tissue for transplantation. If raphe neurons were present within the transplants, they would be visible in sections reacted immunocytochemically for serotonin. We have never observed any such neurons in our transplants in this or other studies, although the serotonergic neurons are routinely visible in brainstem sections processed concurrently.

Synthetic dura (durafilm, Codman-Schurtleff, Inc.) and $0.9 \%$ salinesoaked gelfoam $\left(2 \mathrm{~mm}^{3}\right)$ were placed over the lesion cavity or lesion cavity plus transplant. The overlying muscle and skin were sutured in layers with 6.0 silk. Before being returned to their mother, pups received prophylactic doses of Bicillin (Wyeth Laboratories, Philadelphia, PA) and recovered in a warm environment. Orthograde and retrograde tracing and immunohistochemical techniques were used to examine axonal growth in normal and experimental animals at 1.5-4 months of age. Although the initial spinal cord lesions and transplants were made in newborn animals, the neuroanatomical tracing experiments were performed after these animals had reached maturity (1.5-4 months of age). Thus, we have examined the long-term neuroanatomical reorganization that persisted in the adult animal after neonatal spinal cord injury.

\section{Neuroanatomical tracing paradigm}

Various neuroanatomical tracing techniques were used to examine axonal growth of the following pathways into the injured spinal cord: corticospinal, brainstem-spinal (red nucleus, raphe nuclei, medullary reticular formation), propriospinal and motor neurons, and dorsal root projections. Rats from each experimental group ( $\mathrm{HX}, \mathrm{HX}+\mathrm{TP}, \mathrm{CON})$ were randomly assigned to particular tracing protocols. The distribution of animals within each tracing protocol is illustrated in Table 1. Rats with transplants, lesion alone, or controls were anesthetized with chloral hydrate $(400 \mathrm{mg} / \mathrm{kg}$, i.p.). Rats were shaved and cleaned with $70 \%$ alcohol. Unless noted otherwise below, after application of the neuroanatomical tracer into the spinal cord, durafilm followed by $0.9 \%$ salinesoaked gelfoam was placed over the site of administration. The muscles were sutured with 6.0 silk, and the skin was stapled with $9 \mathrm{~mm}$ stainless steel autoclips. Postoperative survival times are described below.

Orthograde tracing of corticospinal axons. The location of the corticospinal axons in the spinal cord was evaluated in adult CON $(n=4)$, HX $(n=6)$, and $\mathrm{HX}+\mathrm{TP}(n=11)$ rats. Animals were prepared for injections similar to that described previously (Bregman et al., 1989), and details are summarized here. The skin and cranium overlying the sensorimotor cortex were opened. A Hamilton syringe was used to inject 3-5 $\mu \mathrm{l}$ of $10 \%$ FluoroRuby (10,000 molecular weight, in $0.9 \%$ saline; Molecular Probes, Eugene, OR) into the cortex bilaterally. Dry gelfoam was placed over each injection site. Rats were euthanized 10-14 d postinjection, and brain and spinal cord segments were blocked and cryoprotected in a graded series of sucrose solutions.

Retrograde tracing using gelfoam pledgets soaked with fluorescent tracers. To label the cell bodies of neurons projecting into the spinal cord, gelfoam pledgets soaked in $1-2 \mu \mathrm{l}$ of $2 \%$ Fast Blue (FB) [Dr. Illing Plastics, Bergfeld, Germany; postnatal day (P) 3-14 animals] or 4\% FluoroGold (FG) (Fluorochrome Inc., Englewood, CO; P14 to 4 month old animals) in $0.9 \%$ saline were dried and inserted into the spinal cord dorsal root entry zone at C6/C7 or L1/L2. Rats were euthanized 5 d later to allow for sufficient transport of the tracer. For each animal, spinal cord sections between the tracer application and the transplant were analyzed to ensure that tracer had not diffused into or rostral to the transplants. In most animals, the rostrocaudal tracer diffusion was less than one segment. After application of the tracer via the dorsal root entry zone, label was visible across the entire transverse extent of the cord.

Retrograde labeling of brainstem and cortical neurons projecting axons into the spinal cord. Gelfoam pledgets soaked with $4 \%$ FG were inserted into the cervical or lumbar enlargement at the dorsal root entry zone to determine the effect of lesion or lesion plus transplant on the remodeling of supraspinal projections to the cord. A skin incision was made in adult rats above the lower cervical (C7/8: $n=3, \mathrm{CON} ; n=5, \mathrm{HX} ; n=7$, HX $+\mathrm{TP})$ or upper lumbar (L1/2: $n=7, \mathrm{CON} ; n=8, \mathrm{HX} ; n=9, \mathrm{HX}+\mathrm{TP})$ spinal cord. Rats were euthanized $5 \mathrm{~d}$ after application of FG.

Retrograde labeling of propriospinal neurons to examine their developmental appearance and their potential recovery after spinal cord injury. The extent of propriospinal neuronal development at birth in the rat is not known, although these neurons are present during embryonic development of some other species (Oppenheim et al., 1989; Berkowitz and Stein, 1994). Gelfoam pledgets soaked with a fluorescent tracer were placed into the dorsal root entry zone of segments of lumbar spinal cord in normal rats at $3 \mathrm{~d}$ postnatal $(n=10, \mathrm{FB}$; anesthetized by hypothermia), 2 weeks postnatal ( $n=12, \mathrm{FG}$, and $n=4, \mathrm{FB}$, anesthetized with chloral hydrate, $400 \mathrm{mg} / \mathrm{kg}$, i.p.), and as adults (lumbar $n=7, \mathrm{FG}$ ). The spinal cords of experimental adult animals were labeled with FG $(n=8$, $\mathrm{HX} ; n=9, \mathrm{HX}+\mathrm{TP})$ and compared with normal adults.

\section{Perfusions and tissue preparation}

Rats were euthanized by an overdose of chloral hydrate $(1000 \mathrm{mg} / \mathrm{kg}$, i.p.) and perfused intracardially with $0.9 \%$ heparinized saline followed by $4 \%$ paraformaldehyde in $0.1 \mathrm{~m}$ phosphate buffer, $\mathrm{pH}$ 7.4. Spinal cords and brains were dissected and post-fixed in $4 \%$ paraformaldehyde at room temperature for $2 \mathrm{hr}$, followed by cryoprotection in a graded series of sucrose $(10 \%-30 \%)$ in $0.1 \mathrm{M}$ phosphate buffer at $4^{\circ} \mathrm{C}$. Brains and cervical spinal cords were blocked separately, placed in OCT compound (Miles, Elkhart, IN), and frozen on dry ice. Spinal cord tissue was cut in serial horizontal or transverse $16 \mu \mathrm{m}$ sections and thaw-mounted onto gelatin-chrom alum-subbed slides. The brains and brainstems were sectioned at $20 \mu \mathrm{m}$ in the coronal plane in a 1:3 series. 
Table 1. Chart indicating the distribution of anatomical tracing and labeling experiments conducted for each animal in this study

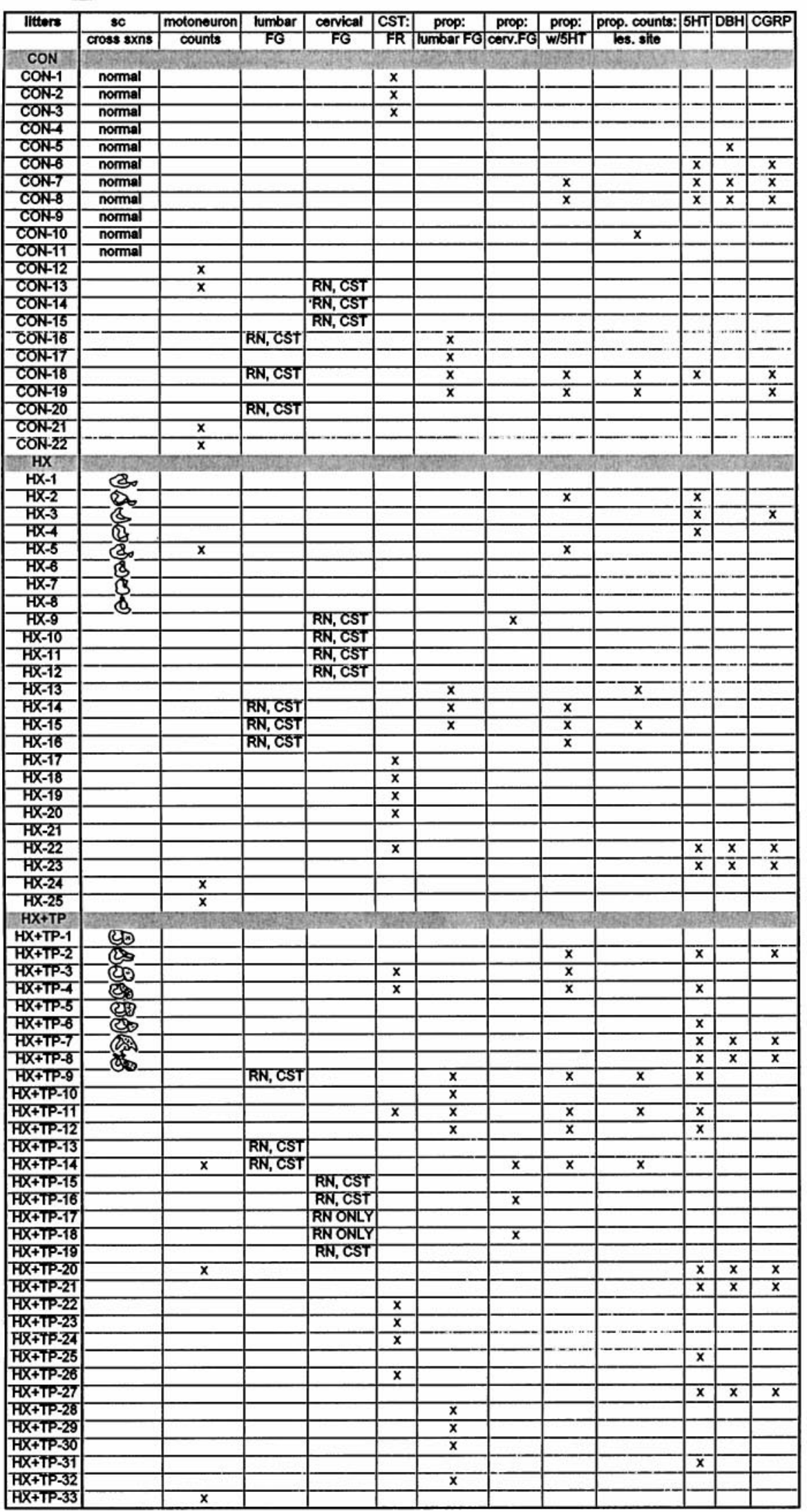

Animals used for behavioral analysis in the companion paper (Diener and Bregman, 1998) are indicated by a drawing of the lesion or lesion plus transplant, or are listed as normal, in the column labeled cross sections (cross sxns). 


\section{Immunocytochemistry}

Peroxidase-antiperoxidase immunocytochemical techniques (Sternberger, 1976) were used to detect immunoreactivity for serotonin [5-hydroxytryptamine (5-HT)]. Avidin-biotin immunocytochemical techniques were used to visualize dopamine $\beta$-hydroxylase and calcitonin gene-related peptide immunoreactive processes in spinal cord sections adjacent to those stained with cresyl violet. These procedures have been described in detail elsewhere (Sternberger, 1976; Bregman, 1987a,b; Jakeman et al., 1989; Bregman et al., 1991; Jakeman and Reier, 1991).

Serotonin. Antibodies against serotonin were used to visualize raphespinal projections (CON, $n=4$; $\mathrm{HX}, n=8$; $\mathrm{HX}+\mathrm{TP}, n=12$ ) within the host cord and transplant. Spinal cord tissue was incubated overnight at room temperature in primary antibody [rabbit anti-serotonin-bovine serum albumin conjugate (Incstar, Stillwater, MN); 1:3000 with $0.3 \%$ Triton X-100 in PBS and 5\% normal goat serum (PBS-NGS)]. Sections were washed and incubated for $45 \mathrm{~min}$ with the secondary antibody, goat anti-rabbit IgG (Sternberger Monoclonals, Baltimore, MD), at a 1:10 dilution in PBS-NGS. Sections were rinsed and incubated for $45 \mathrm{~min}$ in rabbit peroxidase-antiperoxidase (Sternberger Monoclonals) at a dilution of 1:200. The reaction product was visualized with diaminobenzidine (DAB) with nickel intensification. Slides were rinsed, dehydrated, cleared in xylene, and coverslipped with permount.

To identify potential associations between raphespinal axons and propriospinal neurons, the serotonin immunocytochemistry protocol was repeated with some modifications. Spinal cord tissue (C5 segment) from animals that received FG administration to lumbar segments (refer to neuroanatomical tracing paradigm above: $\mathrm{CON}, n=4 ; \mathrm{HX}, n=3$; $\mathrm{HX}$ $+\mathrm{TP}, n=4)$ was used. We deviated from the serotonin protocol described above at the secondary antibody incubation stage by using an IgG conjugated to fluorescein (FITC-labeled IgG; Hyclone Labs, Logan, UT). After the $45 \mathrm{~min}$ incubation with the secondary antibody at room temperature, the slides were washed, coverslipped (mounting media, Citifluor), and examined and photographed immediately with a Zeiss microscope (excitation filter at $546 \mathrm{~nm}$ ).

Calcitonin gene-related peptide (CGRP) or dopamine $\beta$-hydroxylase $(D B H)$. Spinal cord segments adjacent to those used for serotonin immunoreactivity were incubated for $2 \mathrm{~d}\left(4^{\circ} \mathrm{C}\right)$ in either the primary antibody against CGRP (dilution of 1:5000; Peninsula Labs, Belmont, CA) (CON, $n=3$; $\mathrm{HX}, n=3 ; \mathrm{HX}+\mathrm{TP}, n=4)$ to label a subpopulation of dorsal root axons or the primary antibody against DBH (dilution of 1:1200; Eugene Tech Labs, Allendale, NJ) (HX, $n=2 ; \mathrm{HX}+\mathrm{TP}, n=$ 7) to label noradrenergic axons in the spinal cord. Sections were washed and incubated at room temperature for $1 \mathrm{hr}$ with the secondary antibody (Vectastain ABC Kit; Vector Laboratories, Burlingame, CA). Sections were rinsed and exposed to avidin and a biotinylated enzyme (Vectastain $\mathrm{ABC}$ Kit, Vector) and then washed and reacted with peroxidase and $\mathrm{DAB}$ to reveal a brown reaction product. Sections were dehydrated in $100 \%$ alcohol, cleared in xylene, and coverslipped.

\section{Morphometry}

Serial transverse $16 \mu \mathrm{m}$ cryostat sections (1:5 series) through the lesion site were stained with cresyl violet and serially reconstructed using an aus Jena microprojector and a Zeiss microscope. Reconstructed sections were used to examine the rostrocaudal and transverse extent of both the lesion site and the transplant apposition and to verify the absence of an obvious glial boundary (Reier et al., 1986) between the host and transplant. This method identified those animals that met the lesion criteria. Although we did not use electron microscopy or immunocytochemistry to visualize the glia in this study, the cellular appearance at the hosttransplant interface was identical to that observed in our earlier studies (Bregman and Reier, 1986; Reier et al., 1986) and lacked an obvious cellular barrier between host and transplant tissue.

Lesion sites from each rat were evaluated for completeness of the injury. A complete lesion severed the entire dorsal funiculus, the right lateral and ventral funiculi, and the intervening gray matter represented in Figure $1 A, B$. Criteria were established for maximum and minimum extent of the lesion site as shown in Figure $1 B$. The lesion plus transplant group had the additional criteria that the transplant was present and in continuous apposition to the host cord without an intervening cellular barrier (Fig. 1C). Sections were stained with cresyl violet to aid in identifying characteristic histology of the transplant and host cord. Only rats meeting the lesion criteria were used in this study and were assigned randomly to groups for immunocytochemistry (see Table 1). Animals used in fluorescent tracer studies had the additional requirement that the tracer must not have spread beyond the insertion site or leaked into the
CSF (spinal cord and cortex injections). The animals that had insufficient bilateral uptake of the tracer were excluded from further analysis.

Tissue sections were analyzed with a Zeiss Axioscope microscope to identify propriospinal, raphespinal, rubrospinal, and corticospinal pathways: sections were viewed with (1) bright-field illumination for cresyl violet staining or immunohistochemistry using DAB as the chromagen or (2) fluorescence illumination with excitation filters at $365 \mathrm{~nm}$ for FluoroGold, $485 \mathrm{~nm}$ for FluoroRuby, or $546 \mathrm{~nm}$ for the fluorescein label. Cresyl violet-stained brainstem (1:3 series) sections were used for identification of the boundaries of the red nucleus (RN) and for qualitative interpretation of other surviving neuronal populations. Cells retrogradely labeled by FluoroGold identified the neurons in the brainstem and cortex that extended axons into the host cord and/or transplant. The location of labeled neurons within the RN, raphe nuclei, and layer V of the sensorimotor cortex were mapped on standard brain slices by examining each section through the region of interest. Representative sections from each region were photographed. RN neurons throughout the rostrocaudal extent of the nucleus also were assessed quantitatively (i.e., cell counts) after both cervical and lumbar injections (see below). The course of the corticospinal axons anterogradely labeled with FluoroRuby were examined in representative coronal sections throughout the brain and brainstem and cross sections throughout the spinal cord.

\section{Quantitative assessments of neuronal populations}

At least three animals in each group that had robust retrograde filling of neurons were selected for further quantitative analysis. Within each group, these animals were matched for cross-sectional extent of lesion, extent of transplant, and degree of apposition.

$R N$ cell counts. The number of RN neurons that retrogradely transported FluoroGold from cervical (CON, $n=3$; HX, $n=3$; HX + TP, $n=3$ ) or lumbar (CON, $n=3$; HX, $n=3$; $\mathrm{HX}+\mathrm{TP}, n=3$ ) spinal cord were quantified by counting all FluoroGold-labeled neurons with a nucleus in the plane of section from every fifth section throughout the rostrocaudal extent of the red nucleus (Zeiss microscope; excitation filter, $365 \mathrm{~nm}$ ). The number of neurons present in each operated group was compared with control numbers to determine the percentage of neurons spared after hemisection or hemisection plus transplantation. The position of the individual neurons within the nucleus in each section was recorded with regard to dorsal, ventral, and mediolateral planes.

Propriospinal neurons with associated serotonergic axons. The C5 spinal cord segment was cut in serial transverse sections at $16 \mu \mathrm{m}$. All spinal cord sections were examined for the presence of FluoroGold-labeled propriospinal neurons (Zeiss Axioscope microscope; excitation filter, $365 \mathrm{~nm}$ ). Five to six randomly chosen tissue sections from the first third of the C5 series per animal ( $\mathrm{CON}, n=3$; $\mathrm{HX}, n=3$; $\mathrm{HX}+\mathrm{TP}, n=4)$ were used to count propriospinal neurons in laminae VII-VIII bilaterally. Identified propriospinal neurons were examined for the presence of serotonergic-immunofluorescent fibers (Zeiss Axioscope microscope; excitation filter, $546 \mathrm{~nm}$ ) traversing along or over the contours of the soma or proximal dendrites. For the purposes of quantification, the percentage of propriospinal neurons with serotonin-immunofluorescent fibers that were adjacent to, apposed to, or associated with the propriospinal neuron was determined. Propriospinal neurons with descending associations were compared with the total number of propriospinal neurons present in laminae VII-VIII for each examined animal. The final ratio was expressed as a percentage of the change in the number of propriospinal neurons with associated serotonergic axons in the operated groups as compared with controls.

Ventral motoneurons. The C5 spinal cord segment $(\mathrm{CON}, n=3 ; \mathrm{HX}$, $n=3$; HX $+\mathrm{TP}, n=3$ ) was blocked and cut in transverse serial sections at $16 \mu \mathrm{m}$. This segment was chosen because it contains motor neurons controlling forelimb muscles and is the first brachial segment caudal to the lesion site. If secondary effects of the spinal injury were to interfere with forelimb motoneuron survival, then changes would be pronounced at the segment immediately caudal to the lesion. A 1:3 series of sections from the rostral, intermediate, and caudal third of the C5 spinal cord segment from each animal were stained with cresyl violet and viewed in bright-field illumination with a Zeiss Axioscope microscope equipped with a drawing tube to count the number of motoneurons with visible nuclei in laminae IX, bilaterally. From a random starting point in each region of the C5 segment, 10 sections per animal were counted. Total numbers from each operated group were recorded and compared with the total number of motoneurons in the control group to determine whether the absence of forelimb target-reaching was correlated to the number of motoneurons remaining in the cervical enlargement. 


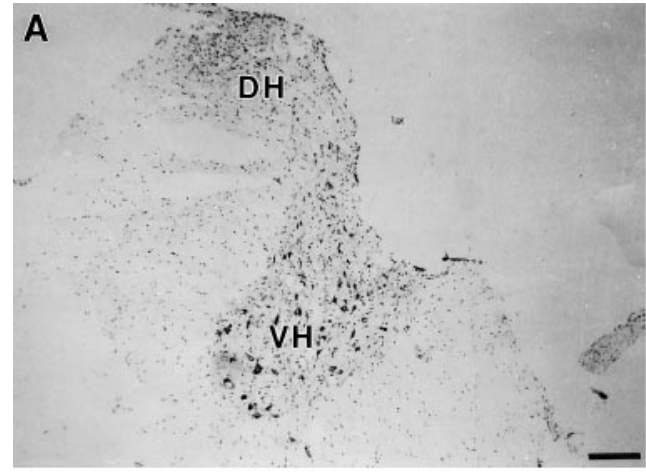

B
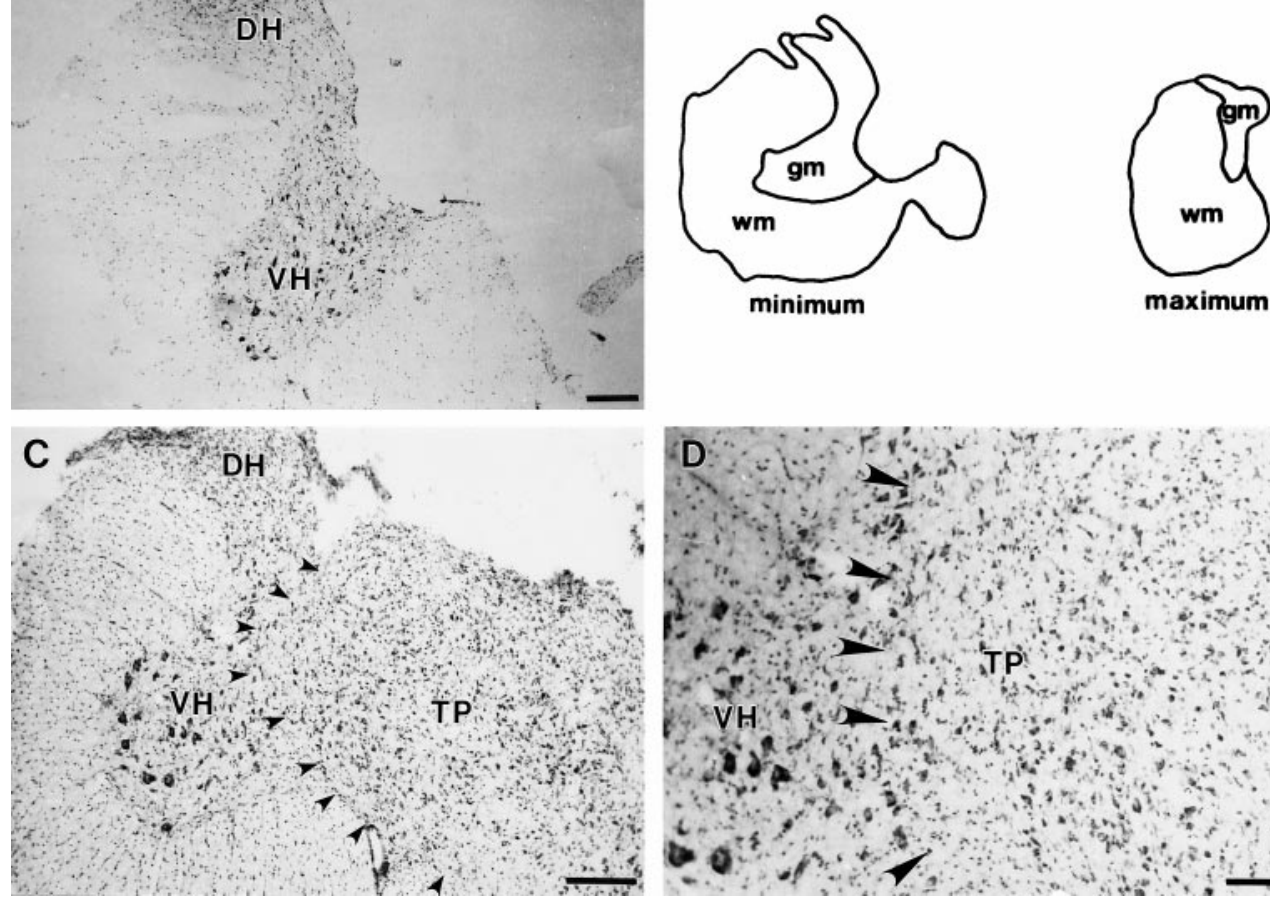

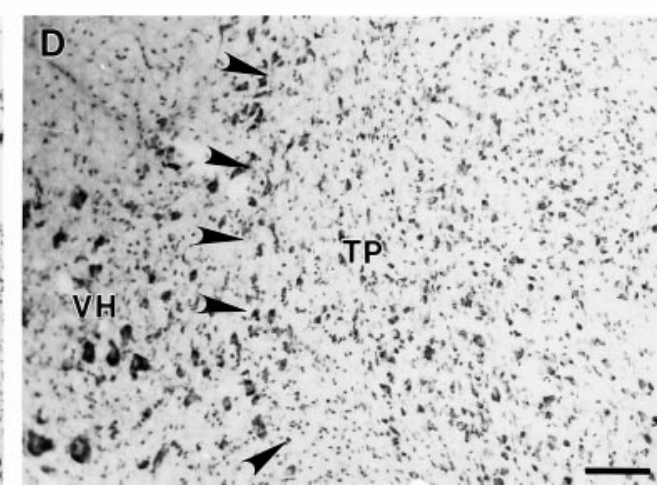

Figure 1. Segments of cervical spinal cord through the lesion and lesion plus transplant site. $A, C, D$, Representative spinal cord cross sections stained with cresyl violet. These sections demonstrate the transverse extent of the lesion (or lesion plus transplant) in animals meeting the inclusion criteria for this study (see Materials and Methods). Spinal cords of both HX and HX + TP animals were completely overhemisected, as shown in $A$ and $C$ (i.e., typical overhemisections included damage to the right side of the cord and bilateral ablation of the dorsal funiculus). $B$, The tracings are representative sections through the lesion site of different HX animals to depict the minimal and maximal extent of injury acceptable for this study, as described in the criteria for lesion section (see text). $C$, Photomicrograph of a representative section through the lesion plus transplant region demonstrating the transverse extent of the apposition of the transplant to the host cord (small arrowheads). $D$, High-power view of the interface between host and transplant to show (1) that the transplant grew to fill the lesion cavity and (2) the close apposition (large arrowheads) of transplant and host tissue without an intervening cellular barrier. Scale bars: $A, C, 100 \mu \mathrm{m} ; D, 50$ $\mu \mathrm{m}$. $D H$, Dorsal horn; $T P$, transplant; $w m$, white matter; $g m$, gray matter; $V H$, ventral horn.

\section{Statistics}

The quantitative results from the ventral motoneuron cell counts were analyzed using one-way ANOVA (Sigma Stat, Jandel Scientific, Corte Madera, CA) to determine whether the lesion or lesion plus transplant affected cell survival. The results of the statistical analysis were accepted as significant if $p$ values were $\leq 0.05$. Cell counts were presented as the total number of motoneurons averaged for each group (mean \pm SD).

\section{RESULTS}

\section{Lesion reconstructions}

Thirty-five lesion-only and 33 transplant rats [including the rats used for behavioral analysis in Diener and Bregman (1998); Table 1] met all of the criteria for inclusion in the data analysis. Figure $1 A$ depicts a representative overhemisection that met the lesion criteria. Representative tracings illustrating the acceptable minimum and maximum lesion extents are shown in Figure $1 B$. The lesion ablated the dorsal funiculus bilaterally and the right lateral and ventral funiculi and intervening gray matter unilaterally. The rostrocaudal extent of the lesion was similar (4-5 $\mathrm{mm}$ in length) in lesion-only and lesion plus transplant animals. Embryonic transplants were integrated well with the host spinal cord both transversely and rostrocaudally. The transplants were healthy and morphologically distinct from the host spinal cord and characterized by the smaller size of the neurons and the absence of a distinct segregation of gray and white matter characteristic of the spinal cord (Fig. 1C,D). The transplants were apposed to the host cord, without any obvious intervening cellular barrier.

\section{Brachial motor neurons}

The number of motoneurons in the C5 spinal cord segment was examined in control, lesion-only, and lesion plus transplant groups and matched for the transverse extent of the lesion to determine whether neonatal spinal cord injury caused damage to the motor neuron pool caudal to the lesion site. Qualitatively, the appearance of motoneurons at C5 was similar in each group; healthy motoneurons were readily visible within the ventral horn in all animals (Fig. $2 A, B)$. Quantitative analysis (Fig. $2 C)(p>0.05)$ indicated that the number of motoneurons was similar in control, hemisection, and hemisection plus transplant groups. Thus, the deficits in forelimb use observed in the companion paper (Diener and Bregman, 1998) cannot be attributed directly to loss of motoneurons innervating forelimb muscles. Rather, differences in motor capacity may be related to differences in descending and dorsal root afferent input to the cervical spinal cord.

\section{Axon projections into the transplant}

In all animals with transplants, the transplant was in direct apposition with the host spinal cord, providing an uninterrupted terrain for growing supraspinal and segmental axons to interact with neurons within the transplant. The extent of descending and dorsal root axonal growth was examined by neuroanatomical tracing (corticospinal fibers) and by immunocytochemistry (5HT, noradrenergic, and CGRP containing dorsal root axons). Corticospinal axons labeled anterogradely with FluoroRuby entered the transplant and extended throughout its rostrocaudal and mediolateral extent (Fig. 3A). Dorsal root axons (Fig. $3 B$ ) and brainstem-spinal serotonergic (Fig. $3 C, D$ ) and noradrenergic axons (data not shown) also entered and extended throughout the transplant. Although the serotonergic projection within the transplant was less dense than that in the host, the morphological appearance of the fibers was similar to that in the host in terms of axon diameter, presence of varicosities, and so forth. The presence of host axons within the transplants provides a potential anatomical basis for greater control of forelimb function in transplant compared with lesion-only animals. 

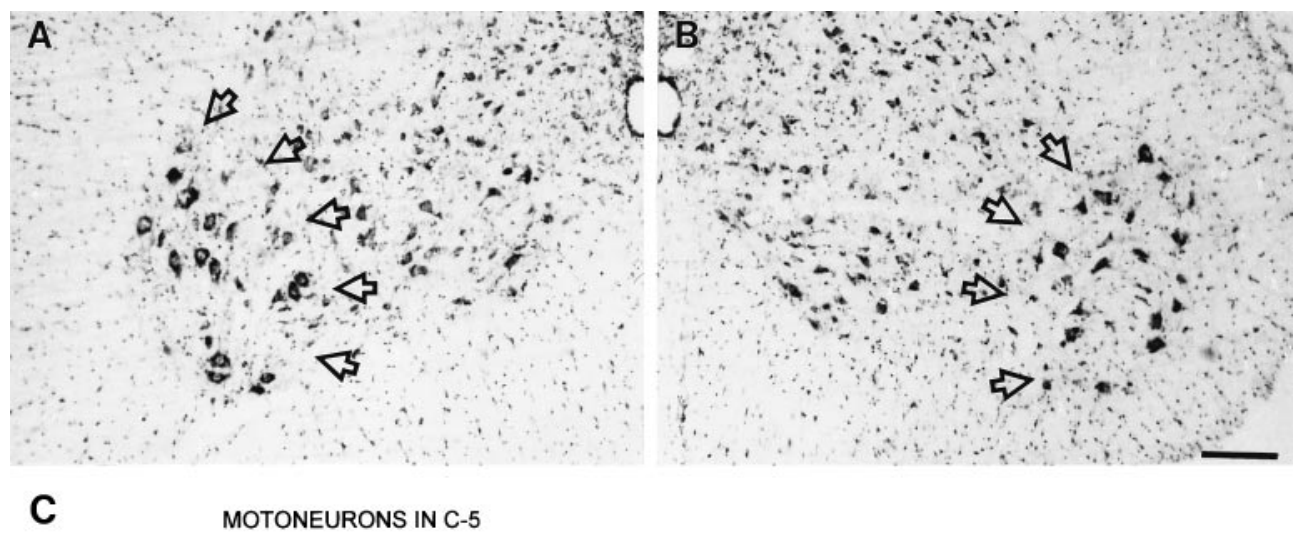

Figure 2. Effect of neonatal cervical spinal cord injury on the motoneuron pool in the brachial spinal cord segment C5. $A, B$, High-power view of both ventral horns from a representative cresyl violet-stained cross section through a $\mathrm{C} 5$ segment from an HX animal. The photomicrographs show that a healthyappearing motoneuron pool in laminae IX (delineated by open arrows) is present. Scale bar, $50 \mu \mathrm{m}$. $C$, Histogram showing that the total number of motoneurons present in laminae IX of each group's left and right ventral horn is similar (CON, $n=3 ; H X, n=3 ; H X+$ $T P, n=3)$. Error bars represent SD; $p>$ 0.05 .

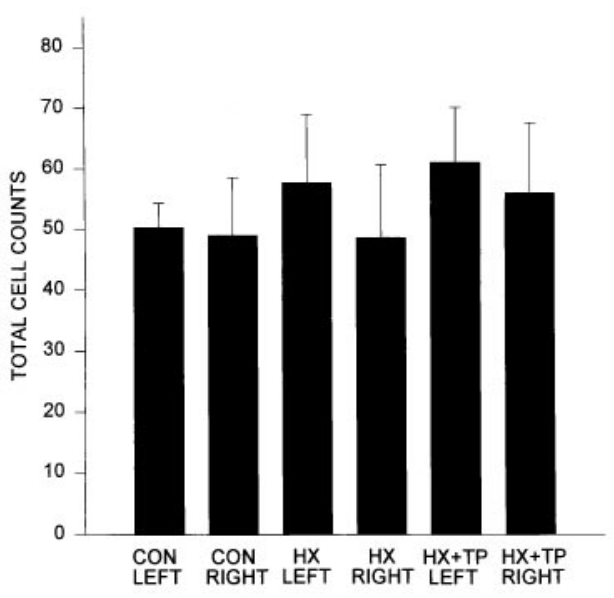

\section{Retrograde labeling of neurons Transplant}

Not only did host neurons project within the transplant, but also neurons within the transplant projected axons into the host spinal cord. In many cases the transplanted neurons extended axons long distances within the host spinal cord. FluoroGold inserted into either the cervical (C6-C7) or lumbar (L1-L2) spinal cord (Fig. $3 E, F)$ retrogradely labeled neurons within the transplant in five out of a total of eight animals examined. Morphologically, all transplant neurons retrogradely labeled with FluoroGold were small and round and typically were distributed throughout the rostrocaudal and mediolateral extent of the transplant. Surprisingly, the number of labeled neurons in the transplant after lower cervical injections was not consistently higher than after upper lumbar injections, despite the closer proximity of the site of injection to the transplant.

\section{Cortex}

After tracer application to upper lumbar spinal cord, corticospinal neurons were labeled bilaterally and symmetrically in control (unlesioned) rats (Fig. 4A,B). The continuous band of retrogradely labeled corticospinal neurons extended from $\sim 0.26 \mathrm{~mm}$ to $3.30 \mathrm{~mm}$ caudal to bregma. At its greatest extent, the mediolateral distribution was $\sim 1 \mathrm{~mm}$. In all groups, neurons labeled by tracer administration to the cervical spinal cord were located within the standard forelimb and hindlimb regions of layer $\mathrm{V}$ of the sensorimotor cortex (i.e., $0.48 \mathrm{~mm}$ rostral to $2.80 \mathrm{~mm}$ caudal to bregma with a mediolateral extent of $1.5 \mathrm{~mm}$ ). The band of neurons was continuous and densely packed in normal animals (Fig. 5A,B). After cervical hemisection at birth, FluoroGold injected into the lumbar spinal cord rarely labeled corticospinal neurons (Fig. 4C,D). Although retrogradely labeled corticospinal neurons were observed after cervical injection of FluoroGold (Fig. 5C,D), the population was dramatically reduced compared with the normal population of spinally projecting neurons. After cervical labeling, despite the closer proximity of FluoroGold application, total numbers of labeled neurons remained modest in the lesion-only group (compare Fig. $4 C, D$ with Fig. $5 C, D$ ). This injury-induced reduction in labeled neurons was particularly evident in the cortex contralateral to the lesion. In contrast to lesion-only animals, fetal spinal cord transplants at the lesion site supported the projection of corticospinal axons to both the cervical (Fig. 5E,F) and lumbar spinal cord (Fig. 4E,F).

There were more retrogradely labeled neurons in all lesion plus transplant animals than there were in lesion-only animals. After FluoroGold injection at the cervical level, in transplant animals there was a marked increase in bilateral labeling of corticospinal neurons (Fig. 5E,F) compared with both the lesion-only group (Fig. 5C,D) and the transplant animals after lumbar tracer application (Fig. 4E,F). Differences in cortical neuronal labeling were also noted within the cortex of the transplant animals. Labeled corticospinal neurons in transplant animals were typically more numerous in the cortex ipsilateral (Fig. $4 F, 5 F$ ) than contralateral to the surgery site in the spinal cord (Fig. $4 E, 5 E$ ). The corticospinal projection was reduced, however, compared with that in normal control animals.

\section{Red nucleus}

After the insertion of FluoroGold into the lumbar spinal cord, rubrospinal neurons in normal animals were labeled bilaterally and symmetrically and restricted primarily to the ventrolateral division of the nucleus (Fig. 6A). Neuronal labeling within the intact nu- 

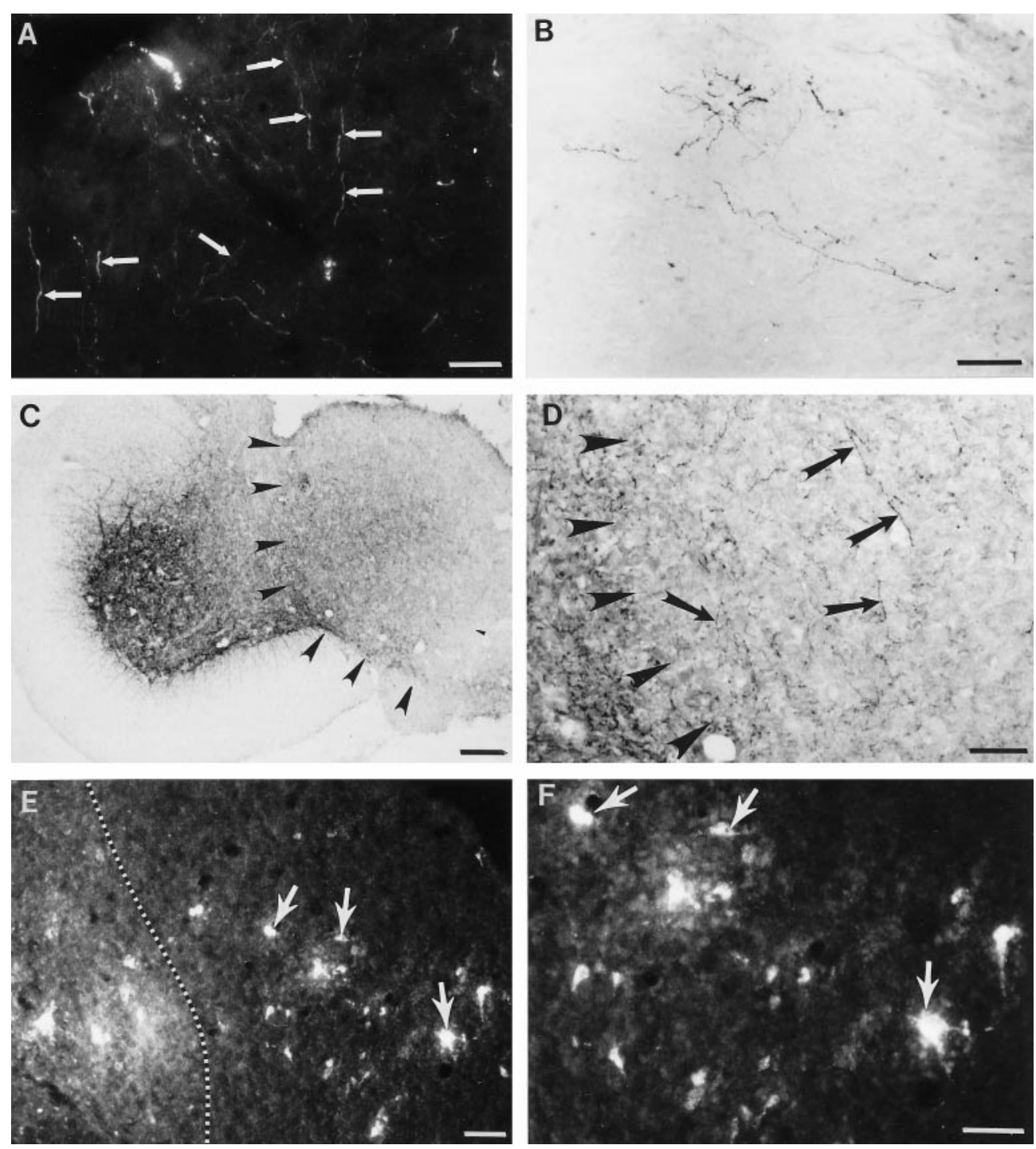

Figure 3. Axonal projections penetrate into and beyond the transplant. $A$, Photomicrograph of FluoroRuby-labeled corticospinal axons (CS) within the transplant. Many CS axons extend throughout the transverse extent of the transplant. $B$, Photomicrograph of a horizontal section through a transplant labeled with antibodies against CGRP. Immunoreactive CGRP dorsal root axons are visualized projecting along the length of the transplant. Individual fibers contain multiple varicosities. $C$, Low-power photomicrograph at the apposition site of the host cord and fetal spinal cord transplant. The interface of the transplant to the host tissue is depicted by arrowheads. Serotonergic axons project robustly throughout the host tissue adjacent to the transplant and within the transplant. $D$, High-power photomicrograph of the transplant at the interface (arrowheads) with the host tissue. Axons immunoreactive for serotonin (arrows) grew within the transplant. Individual axons have many varicosities along their length. E, Low-power photomicrograph of neurons intrinsic to the spinal cord transplant. Neurons were retrogradely labeled with FluoroGold from the lumbar spinal cord. Numerous neurons within the transplant (arrows) are labeled, indicative of their long-distance growth into the host cord. Host-transplant interface is marked with a dashed line. F, Highpower photomicrograph of the identified neurons (arrows) from $E$. Scale bars: $A, B$, $C, E, 50 \mu \mathrm{m} ; D, F, 100 \mu \mathrm{m}$. cleus of both experimental groups resembled this normal topography. Labeling within the axotomized nuclei of both operated groups differed markedly, however. The axotomized red nucleus in the lesion-only animals was devoid of labeled neurons (Fig. 6B). This lack of retrograde labeling in the lesion-only animals was attributable to the retrograde cell death of the injured neurons. Examination of adjacent cresyl violet-stained sections confirmed the massive retrograde cell death of the immature axotomized rubrospinal neurons that has been described previously (Bregman and Reier, 1986; Diener and Bregman, 1994). Conversely, in the transplant animals, labeled neurons were apparent, and the ventrolateral topography was maintained in the axotomized red nucleus of transplant animals (Fig. 6C). Adjacent cresyl violet-stained sections confirmed the rescuing of the rubrospinal neurons (Bregman and Reier, 1986; our unpublished data). The retrograde labeling of red nucleus neurons after lumbar application of FluoroGold represents long-distance growth of rubrospinal axons after cervical spinal cord injury and transplantation.

The application of FluoroGold to spinal cord segments C6/C7 allowed for retrograde transport and identification of rubrospinal neurons that extended axons to or beyond cervical spinal cord segments. The normal topography (e.g., neurons located dorsomedially and ventrolaterally within the red nucleus) was pre- served in intact red nuclei in both lesion-only and transplant animals (data not shown). The axotomized red nuclei from lesion-only rats were devoid of labeled neurons (data not shown), whereas those from all transplant rats contained labeled neurons primarily in the dorsomedial region of the nucleus (Fig. 6D).

The total number of labeled cells within the red nucleus from control and the intact red nucleus from operated animals was similar. Cell counts from axotomized red nuclei, in contrast, revealed distinct differences (Table 2). Axotomized red nuclei from both of the operated groups lacked their normal complement of neurons. This was most pronounced in lesion-only animals. For example, $\sim 1 \%$ of the normal population of rubrospinal neurons [i.e., retrograde labeling from lumbar $(0.7 \%)$ or cervical (1\%) spinal cord] extended axons beyond the lesion site. In the presence of a transplant, a larger percentage of rubrospinal neurons from the axotomized red nucleus extended axons to spinal cord segments caudal to the lesion plus transplant site (i.e., $4 \%$ of normal population of rubrospinal neurons labeled after lumbar and $2 \%$ after cervical tracer application) (Table 2).

\section{Raphe and reticular nuclei}

Neurons from the raphe magnus, pallidus and obscurus, and gigantocellularis were labeled bilaterally and symmetrically in 

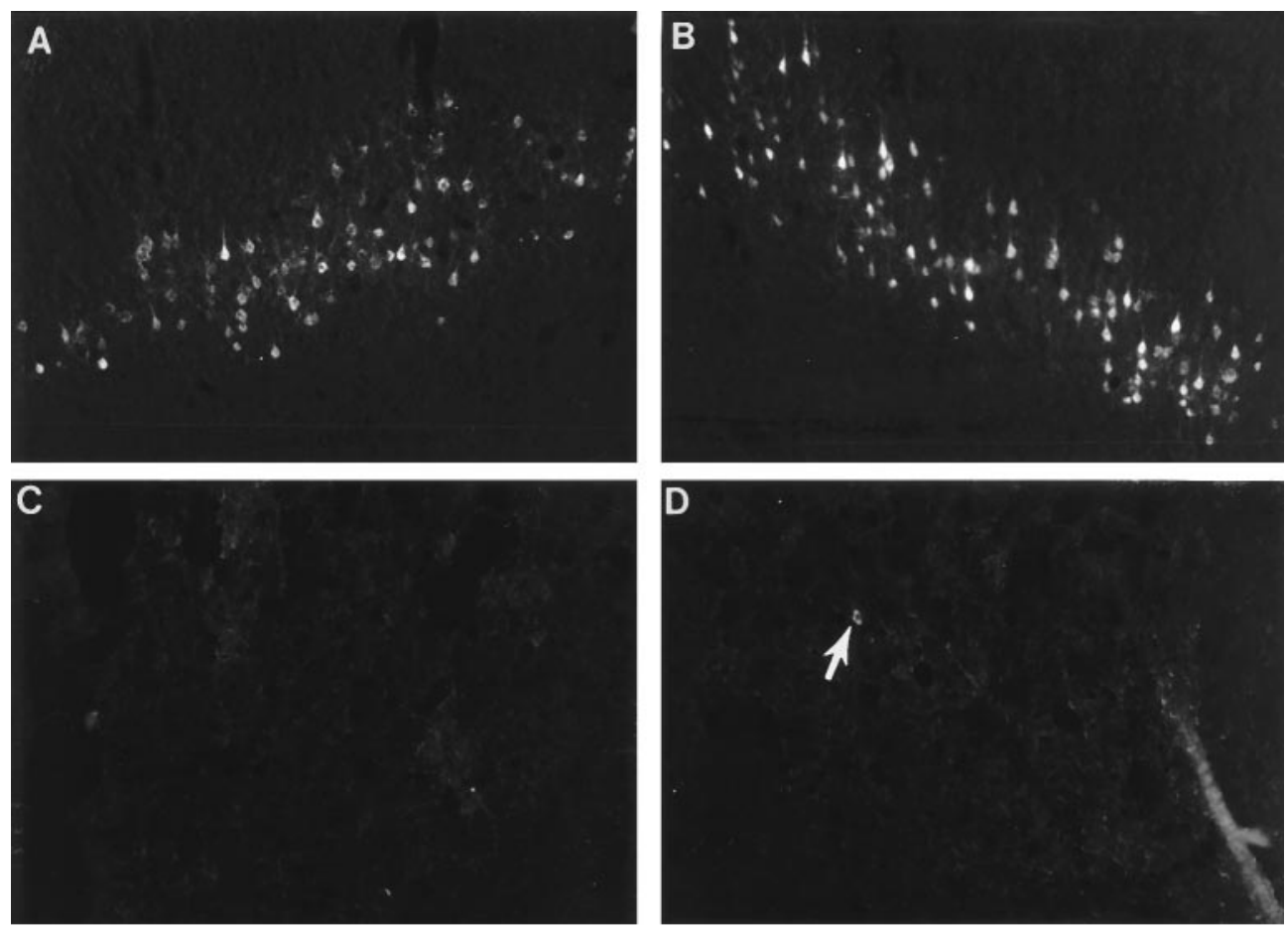

Figure 4. Retrograde tracing of corticospinal neurons from the lumbar spinal cord. In control animals $(A, B)$, retrogradely labeled corticospinal neurons were located in layer $\mathrm{V}$ of the sensorimotor cortex bilaterally. After neonatal hemisection, the number of neurons labeled is reduced in both the contralateral $(C$; devoid of labeled cells) and ipsilateral $(D$; arrow indicating labeled cell) cortices. $E$, $F$, Transplantation spares some of the neurons in both the contralateral $(E ; 3-4$ small cells denoted by arrows) and ipsilateral ( $F$; numerous white cells) cortex. The cortex ipsilateral to the spinal cord lesion $(F)$ has more labeled cells than the contralateral cortex $(E)$. Scale bar (shown in $F$ for $A-F): 100 \mu \mathrm{m}$.
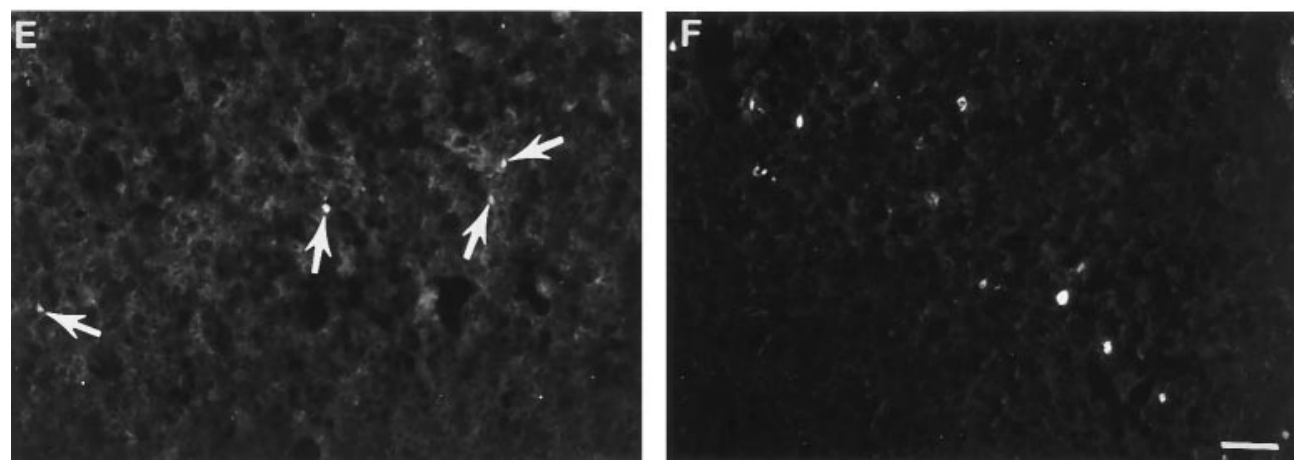

normal animals after application of FluoroGold to the lumbar or cervical spinal cord. Medullary raphespinal neurons in lesiononly animals were labeled asymmetrically (Fig. 7 $A, B$ ). Markedly fewer labeled neurons were present in the raphe magnus and obscurus ipsilateral to the spinal cord injury. In contrast, in transplant animals (Fig. $7 C, D$ ), raphespinal neurons from the raphe obscurus and magnus were labeled bilaterally after tracer application to both cervical and lumbar spinal cord. Labeled reticulospinal neurons were absent typically in the regions adjacent to the midline raphe nuclei in the lesion-only group. Reticulospinal neurons were spared in this region in the transplant group, indicating that the transplant supported growth of raphespinal and reticulospinal neurons to spinal cord sites caudal to the site of transplantation.

\section{Propriospinal neuronal projections in the developing and mature rat spinal cord}

Application of Fast blue (P3) (Fig. 8A) or FluoroGold (P14 and older than P30) to the lumbar spinal cord identified the extent to which propriospinal neurons project into the rat spinal cord. Propriospinal neurons labeled in control animals $(n=18)$ were restricted to specific laminae throughout all cervical spinal cord segments (C2 through C7) that were examined. Primarily, they originated in laminae VII, VIII, and X and were located more sparsely in laminae IV through VI as well as the lateral spinal nucleus (LSN) (Fig. 8B). No retrogradely labeled propriospinal neurons were located in laminae IX.

The laminar location of propriospinal neurons in $\mathrm{C} 2-\mathrm{C} 8$ in the P3 (Fig. $8 A), \mathrm{P} 14(n=19)$, and adult $(>\mathrm{P} 30, n=10)$ normal rats was identical. During development, however, we observed that both the number of neurons within each laminae increased and the morphology became more distinct in the dorsal and ventral horns. At maturity, the propriospinal neurons located within the dorsal horn were smaller and fusiform in morphology, whereas those in the ventral horn were larger and multipolar. Because propriospinal neurons in the cervical cord had developed and extended to the lumbar cord before the third postnatal day, we suggest that axons of propriospinal neurons were severed directly by cervical spinal cord overhemisection at birth. The data from P3 operated animals suggest that neonatal axotomy alters subsequent development of propriospinal neurons within the cervical spinal cord. In sections rostral and caudal to the lesion site in the lesion-only group $(n=5)$, propriospinal neurons were scattered sparsely throughout laminae IV-VIII and X and the LSN. More neurons were labeled in the ventral horn contralateral to the lesion site (Fig. 8C) than in the ipsilateral ventral horn. At the lesion site itself, most sections were devoid of retrogradely labeled propriospinal neurons. 

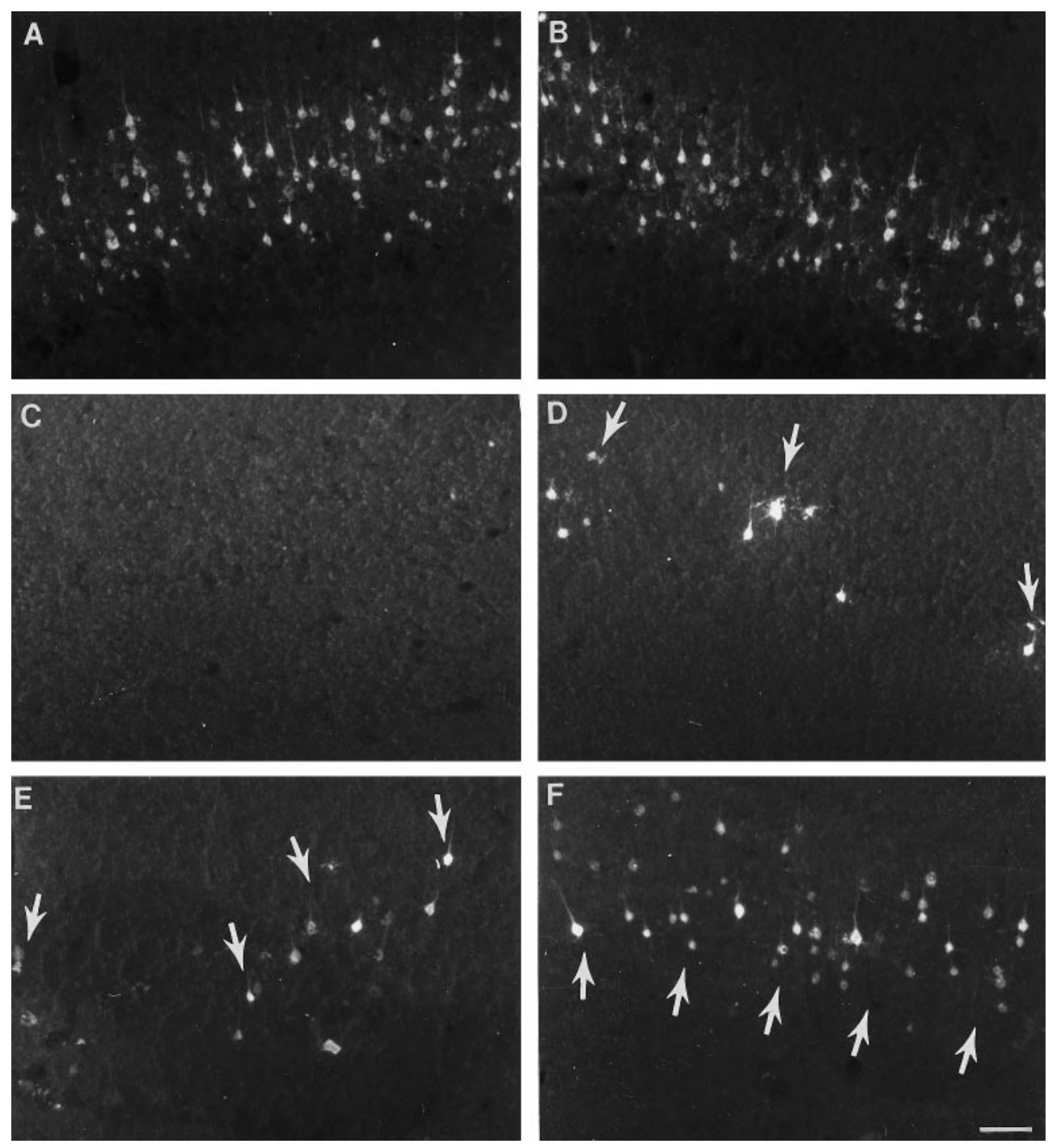

Figure 5. Retrograde tracing of corticospinal neurons from the cervical cord. $A$, $B$, CON cortices showing the normal distribution in layer $\mathrm{V}$ of corticospinal neurons retrogradely traced from cervical spinal cord injections. The mediolateral distribution and the dense packing of cells in laminae $\mathrm{V}$ is greater than after lumbar injections (compare with Fig. $4 A, B)$. After neonatal $\mathrm{HX}$, the number of retrogradely labeled neurons is dramatically reduced in both the contralateral $(C)$ and ipsilateral $(D)$ sensorimotor cortices. In contrast, in all animals with lesion plus transplant, many retrogradely labeled corticospinal neurons are present in the sensorimotor cortex both contralateral $(E)$ and ipsilateral $(F)$ to the spinal cord lesion. There are substantially more retrogradely labeled corticospinal neurons in lesion plus transplant animals $(E, F)$ than in lesion-only animals $(C, D)$. Arrows indicate representative corticospinal neurons labeled retrogradely with FluoroGold. Scale bar (shown in $F$ for $A-F$ ): $100 \mu \mathrm{m}$.
In spinal cord segments both rostral and caudal to the lesion plus transplant site, retrogradely labeled propriospinal neurons were present within laminae IV-V III and X and the LSN, bilaterally (transplant rats, $n=7$ ). Throughout the rostrocaudal extent of the lesion and transplant, labeled propriospinal neurons were identified in the host spinal gray within laminae VII-VIII and often within the LSN. Just caudal to the transplant-host cord interface, labeled neurons in the host spinal cord were present in laminae VII, VIII, and X, and the LSN (Fig. 8D). The transplant, therefore, may support the normal laminar location of propriospinal neurons (illustrated schematically in Fig. 8B) and appears to rescue a substantial proportion of propriospinal neurons bilaterally after early injury to the spinal cord.

\section{Serotonergic axons may contribute to the activity of propriospinal neurons in the cervical spinal cord}

Raphespinal axons projected densely and bilaterally into laminae VII-IX in normal animals. In contrast, the raphespinal projections into the ventral horn (e.g., laminae VIII) immediately adjacent to the lesion were reduced dramatically in lesion-only compared with both normal and transplant animals. In animals with transplants, raphespinal axons extended throughout the ventral horn of the host cord juxtaposed to the transplant, although the projection was reduced slightly compared with that in normal animals. In segments of spinal cord caudal and ipsilateral to the site of transplantation, raphespinal axons arborized throughout the ventral horn (laminae VII-IX).

In normal rats, double-labeling (i.e., FluoroGold and FITC) of the fifth cervical spinal cord segment identified raphespinal fibers (Fig. 9A, green fibers) terminating in close proximity to retrogradely labeled propriospinal neurons (Fig. 9A, white neurons in laminae VIII) within the ventral horn in laminae V II-V III. After hemisection, although propriospinal neurons were labeled, the serotonergic axons projecting to the ventral horn were reduced substantially (Fig. 9B, white neurons and green fibers, respectively). Labeled axons rarely were localized in close association with propriospinal neurons (Fig. 9B; arrow identifies one detectable association). In contrast, both retrogradely labeled propriospinal neurons and densely distributed immunoreactive serotonergic axons were visualized throughout the ventral horn of transplant rats (Fig. 9C, white cells and green fibers in laminae V III). Within laminae VII-VIII, numerous serotonergic axons with varicosities along their length directly apposed the soma and dendrites of propriospinal neurons (Fig. 9C, see arrows; specifically note arrow in center of top edge of photomicrograph and 9D). Thus, the presence of a transplant at the lesion site not only rescues propriospinal neurons after neonatal spinal cord injury, but also the 
Figure 6. Retrograde tracing of rubrospinal neurons after FluoroGold application. The normal pattern of labeling in unlesioned animals is shown in $A$. The retrograde labeling in the intact $\mathrm{RN}$ in $\mathrm{HX}$ and $\mathrm{HX}+\mathrm{TP}$ animals was similar to that in normal animals (data not shown). In contrast, the axotomized RN from HX rats $(B)$ is essentially devoid of labeled neurons after either lumbar $(B)$ or cervical (data not shown) spinal cord lesions. In all animals with transplants at the lesion site, $\mathrm{RN}$ neurons were retrogradely labeled from either the lumbar $(C)$ or cervical $(D)$ spinal cord. Scale bar (shown in $A$ for $A-D$ ): $100 \mu \mathrm{m}$.
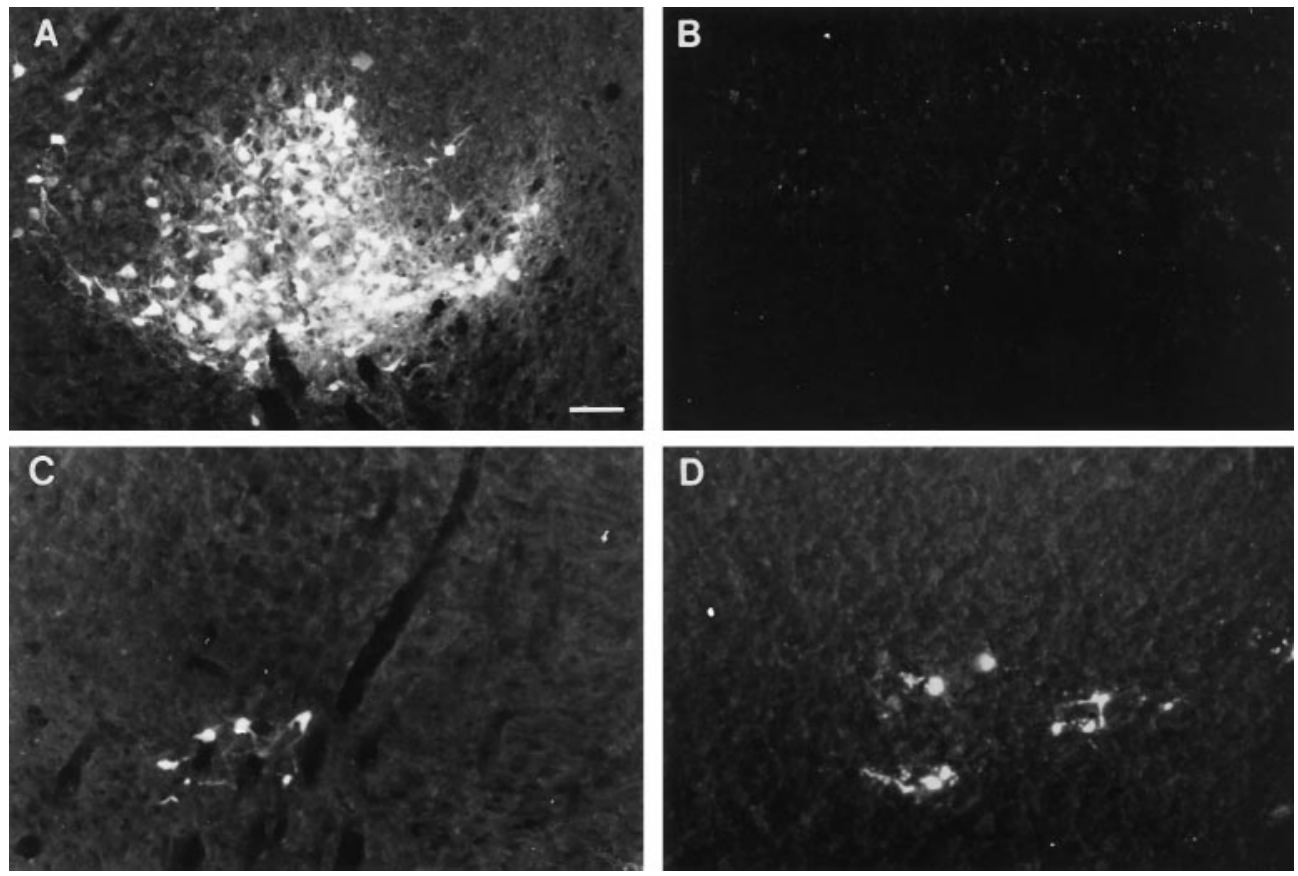

Table 2. Quantitative analysis of red nucleus neurons extending to the lumbar or cervical spinal cord

\begin{tabular}{llll} 
Litter & $\begin{array}{l}\text { Total counts of } \\
\text { rubrospinal } \\
\text { neurons } \\
\text { intact }\end{array}$ & $\begin{array}{l}\text { Total counts of } \\
\text { rubrospinal } \\
\text { neurons } \\
\text { axotomy }\end{array}$ & $\begin{array}{l}\text { Percentage } \\
\text { intact to } \\
\text { axotomy }\end{array}$ \\
\hline $\begin{array}{l}\text { Lumbar injections } \\
\text { CON }\end{array}$ & $755,1275,1637$ & $835,1325,1650$ & \\
HX & $630,900,1700$ & $7,7,8$ & $1,0.7,0.4$ \\
HX + TP & $815,915,920$ & $12,35,60$ & $2,4,6$ \\
Cervical injections & & & \\
CON & 2420,2965 & 3030,2810 & \\
HX & $2290,2580,3340$ & $25,15,45$ & $1,0.6,1.3$ \\
HX + TP & $2470,2650,3435$ & $38,63,70$ & $2.2,1.4,2$ \\
\hline
\end{tabular}

Equivalent numbers of labeled neurons were seen in each $\mathrm{CON}$ rat after lumbar $(n=3)$ and cervical $(n=2)$ injections. One percent or less of the neurons located in the axotomized red nucleus of lesion-only rats extended axons into the spinal cord after lumbar or cervical injections. Approximately $2-4 \%$ of the neurons in the axotomized red nucleus of transplant rats projected axons to the cervical or the lumbar spinal cord, respectively.

descending input to these neurons is reestablished. In addition, serotonergic axons also projected throughout the transplant (Fig. $3 C, D)$, where they may potentially influence intrinsic transplant neurons (Fig. $3 E, F$ ) that project caudally to the lumbar spinal cord.

\section{Quantification of the observed associations between raphespinal axons and propriospinal neurons within laminae VIII of the C5 spinal cord segment}

Representative randomly chosen cross sections of spinal cord from normal, lesion-only, and lesion plus transplant animals were used to quantify the proportion of propriospinal neurons with apposed serotonergic axons. For example, compared with control animals in which all retrogradely labeled propriospinal neurons had serotonergic axons directly apposed, only $69 \%$ of the propriospinal neurons from lesion-only animals were associated with raphespinal axons (i.e., this represents a $31 \%$ reduction in the number of propriospinal neurons associated with serotonergic axons). The brainstem input to the spinal cord was almost completely restored in animals with transplants. In fact, $92.6 \%$ of propriospinal neurons had raphespinal axons apposed (i.e., only $7.4 \%$ of the propriospinal neurons normally associated with serotonergic axons lacked visible relationships).

\section{DISCUSSION}

The companion study (Diener and Bregman, 1998) showed that cervical spinal cord injury delays motor development, impairs postural and limb reflexes, and prevents the development of target-directed reaching. Skilled forelimb movement including target reaching develops in the animals with transplants. The current study was conducted (1) to determine the extent to which fetal spinal cord transplants support axonal elongation after neonatal cervical spinal cord injury and (2) to identify potential neural mechanisms that contribute to the recovery observed. The results suggest that transplantation at the time of injury in the neonate enhances development of forelimb motor control after cervical spinal cord injury by supporting extensive growth of corticospinal and brainstem-spinal projections to normal targets within the spinal cord caudal to the lesion. We suggest that the transplant plays an important role in reestablishing patterns of normal connections (summary schematic shown in Fig. 10) within the injured spinal cord to produce forelimb skilled movements and appropriate postural adjustments. This study suggests that the anatomical growth of particular pathways contributes to the restoration of skilled forelimb movement after cervical spinal cord injury and transplantation.

\section{Remodeling of corticospinal and rubrospinal pathways}

On the basis of the anatomical data in the current and previous studies (Bregman, 1987a,b; Bregman et al., 1989, 1991; Bregman and Bernstein-Goral, 1991; Bernstein-Goral and Bregman, 1993, 1997; Bernstein-Goral et al., 1997; Bregman, 1994), there are several potential mechanisms by which supraspinal input may influence spinal cord neurons (for review, see Bregman, 1994). After neonatal spinal cord injury, the transplant serves as a bridge 

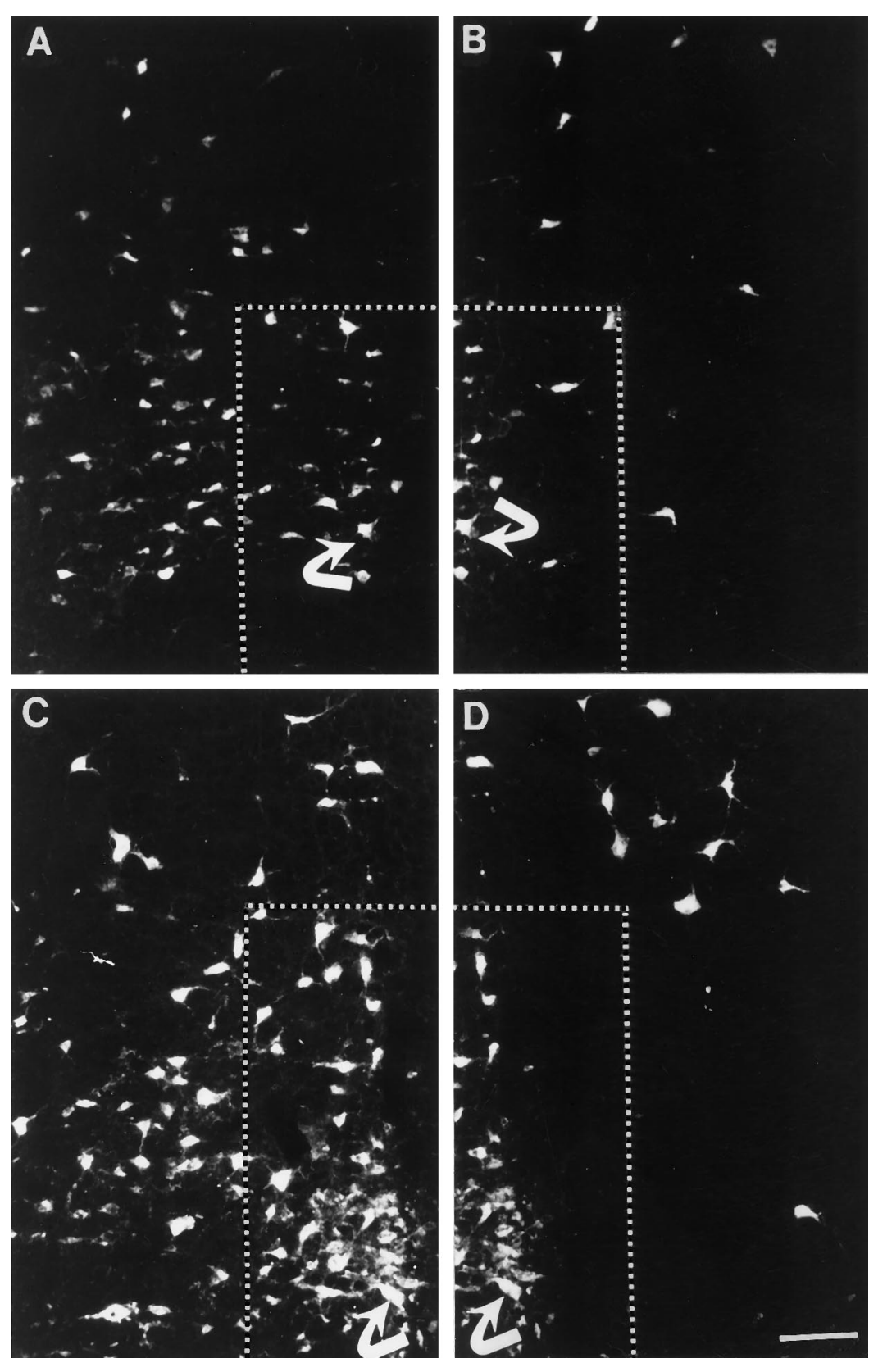

Figure 7. Raphespinal neurons labeled with the retrograde tracer FluoroGold. In each set of panels, the corresponding neuron is identified by a curved arrow for orientation. $A, B$, Photomicrographs of a section from the caudal medulla from a representative HX animal. Diminished labeling is noted in the raphe obscurus, magnus, and pallidus (outlined area, $A-D$ ) on the axotomized $(B)$ side in comparison to the side contralateral $(A)$ to the overhemisection. $C, D$, Photomicrographs of a caudal medullary section from a representative $\mathrm{HX}+\mathrm{TP}$ animal. Labeling varies among transplant animals, but typically a greater number of neurons are labeled in the raphe obscurus and magnus both ipsilateral $(D)$ and contralateral $(C)$ to the spinal cord injury than in HX animals. Scale bar (shown in $D$ for $A-D$ ): $100 \mu \mathrm{m}$. for supraspinal axons to reach both spinal cord levels caudal to the lesion and the transplant directly. In addition, the transplant serves as a relay. Supraspinal neurons project axons into the transplant, and neurons within the transplant project to spinal cord levels caudal to the transplant. Functional synapses form between axons penetrating the transplant and intrinsic transplant neurons (Itoh and Tessler, 1990; Jakeman and Reier, 1991; for review, see Bregman, 1994). It is likely that both regrowth of axotomized pathways and sprouting of undamaged pathways contribute to the lesion-induced plasticity after neonatal spinal cord injury. Transplantation of fetal spinal cord tissue into a neonatal spinal cord lesion site supports the regrowth of axotomized pathways and the remodeling of late-growing spinal pathways (Bregman and Bernstein-Goral, 1991; Bernstein-Goral and Bregman, 1993, 1997; Bernstein-Goral et al., 1997). Both late-developing corticospinal axons and regenerating rubrospinal, coeruleospinal, and raphespinal axons grow long distances beyond a neonatal spinal cord injury and transplant (Bregman and Bernstein-Goral, 1991; Bernstein-Goral and Bregman, 1993, 1997; Bernstein-Goral et al., 1997), suggesting that many of the inhibitory influences that restrict growth after injury in the mature CNS have not yet developed (for review, see Schwab et al., 1993; Schwab and Bartholdi, 1996). Injury in the developing spinal cord also induces compensatory axonal sprouting by axons not directly damaged (for review, see Goldberger and Murray, 1985; Bregman, 1987a; Bernstein-Goral et al., 1997). The anatomical plasticity observed after cervical spinal cord injury and transplantation in the current study may contribute to the development of motor patterns that initiate and execute reaching.

Some of the motor impairments prevalent in the companion study (Diener and Bregman, 1998) resemble those reported by others after rubrospinal and corticospinal tract lesions (Lawrence and Kuypers, 1968a,b; Castro, 1972a,b; Martin and Ghez, 1993) and suggest a similar function of these pathways in the developing 
Figure 8. Retrograde labeling of propriospinal neurons. $A$, In normal animals at P3, propriospinal neurons (arrows) labeled with Fast Blue are distributed throughout laminae VII-VIII and X. This indicates that at the time of the neonatal spinal cord injury, at least some of the propriospinal neurons are axotomized directly. $B$, Schematic diagram representing the general distribution of the propriospinal neurons (represented as stars in LSN, laminae $I V-V I, V I I-$ $V I I I$, and $X$ ) in the brachial cord of a normal adult rat. $C, \mathrm{HX}$ group, spinal cord segment caudal to the injury site. Compared with the normal distribution of propriospinal neurons in adult rats (data not shown), there is a decrease in the number of propriospinal neurons after neonatal hemisection $(C)$. The decrease in labeling is greatest on the side ipsilateral to the lesion but is evident bilaterally. $D, \mathrm{HX}+\mathrm{TP}$ group, spinal cord segment caudal to the lesion plus transplant. In contrast to lesion-only animals, animals with transplants had many retrogradely labeled propriospinal neurons present bilaterally. These neurons were located in all of the appropriate laminae (IV-VIII, X, and LSN). Thus, the transplants rescue a substantial proportion of the propriospinal neurons. Scale bars, $50 \mu \mathrm{m}$. LSN, Lateral spinal nucleus; $C C$, central canal.

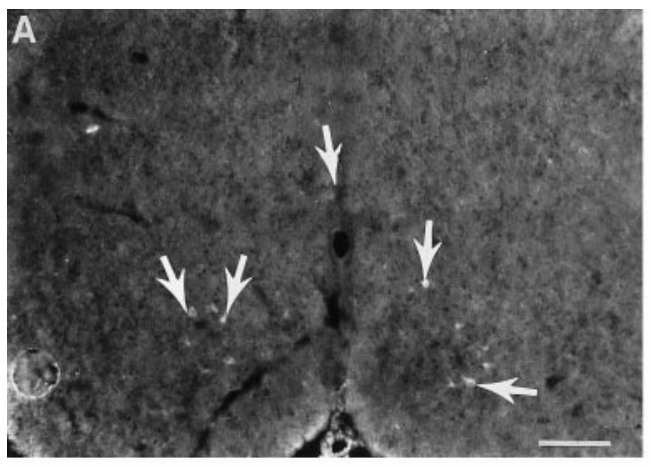

B
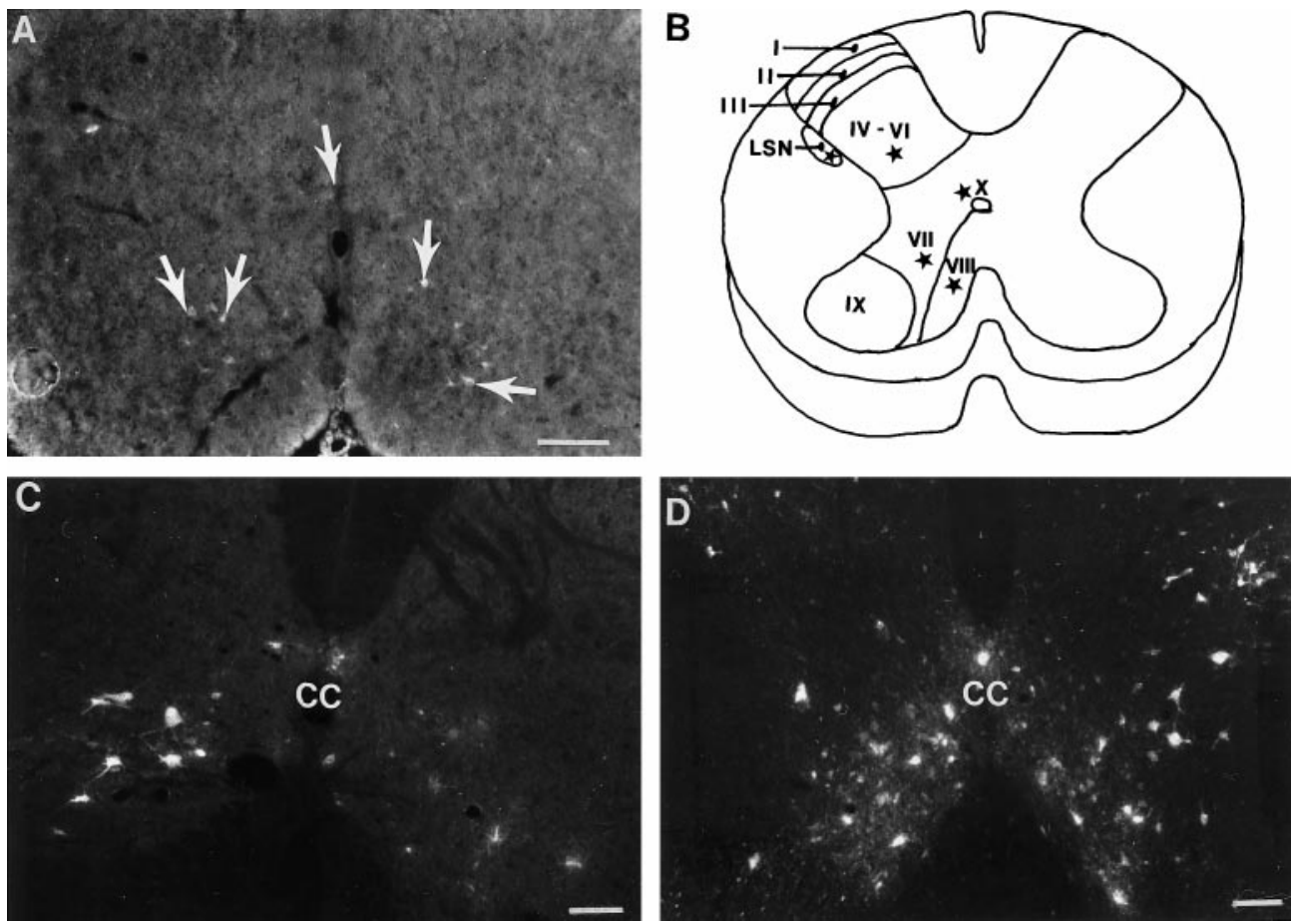

rat. For example, the extent of behavioral recovery after pure pyramidal tract lesions suggests that parallel commands of cortical projections onto rubrospinal neurons may compensate for direct injury to the pyramidal tract. The corticospinal and rubrospinal tracts in the cat (Bernhard and Rexed, 1945; Martin, 1993), monkey (Bernhard and Rexed, 1945), and rat (Waldron, 1969) terminate in the intermediate gray containing interneurons (propriospinals) that project to the motoneurons controlling the distal musculature (Illert et al., 1976b; Illert and Tanaka, 1978; Ghez and Martin, 1982). Combined lesions of pyramidal and rubrospinal tracts result in a decrease in adequate grasping (Evans and Ingram, 1939; Lawrence and Kuypers, 1968b). Reaching and grasping failed to develop in the lesion-only rats; the motor impairment was accompanied by a permanent substantial reduction in the descending projections to the cord caudal to the injury. Rats with transplants develop target-directed reaching, and substantial descending projections are established to the cord caudal to the injury (Diener and Bregman, 1998). Retrograde tracing from either cervical or lumbar spinal cord in transplant rats labeled neurons bilaterally in topographically appropriate regions in both layer $\mathrm{V}$ of the cortex and the red nuclei. This novel finding indicates that corticospinal and rubrospinal axons grow long distances beyond the lesion and transplant. Growth of the supraspinal tracts to brachial cord levels in the presence of the transplant may contribute to the weak but functional grasping patterns used by the transplant rats (Diener and Bregman, 1998). This suggests that descending connections to propriospinal and motoneurons must be reestablished for immature injured rats to develop reaching and grasping.

\section{Remodeling of input to propriospinal neurons}

Neuroanatomical tracing showed numerous raphespinal axons projecting near retrogradely labeled propriospinal neurons in control and transplant animals. The interaction between supraspinal axons and propriospinal neurons within each of the transplant animals was similar in terms of the quantity and extent of association. Moreover, the extent of growth and close proximity of supraspinal axons with propriospinal and other spinal neurons resembles the normal situation qualitatively but not quantitatively. This may be one explanation for why the reaching pattern of transplant rats resembles but is not identical to the normal reaching pattern (Diener and Bregman, 1998).

In the normal cord, a complex mechanism permits targetdirected reaching and keeps all limbs informed regarding body movements and the intent for movement. After injury plus transplants, the major components of reaching are present, but the efficiency, speed, and quality of performance of the skilled movement were often impaired. Short propriospinal neurons receive converging input from supraspinal, segmental and intersegmental projections, and in turn extend collaterals to long propriospinal neurons, other local interneurons, and motoneurons in brachial through lumbar segments (Sterling and Kuypers, 1968; Alstermark et al., 1987b,d). Long propriospinal neurons affect balance during static and dynamic activity through their projections to neurons in the thoracic and lumbar spinal segments (Alstermark and Wessberg, 1985; Alstermark et al., 1987d). Reciprocal connections of neurons originating in the lumbar segments also participate in overall body coordination. Parallel commands that travel from the cortex to the cervical cord also extend directly to neurons in the thoracic and lumbar segments (Alstermark et al., 1981a,b, 1987b, 1990). This allows for direct hindlimb and lower body movements in concert with upper body activity (Alstermark and Wessberg, 1985). Cervical spinal cord hemisection in the neonate disrupts this neuronal pattern. Target-directed forelimb skills fail to develop, and compensatory strategies emerge. The 

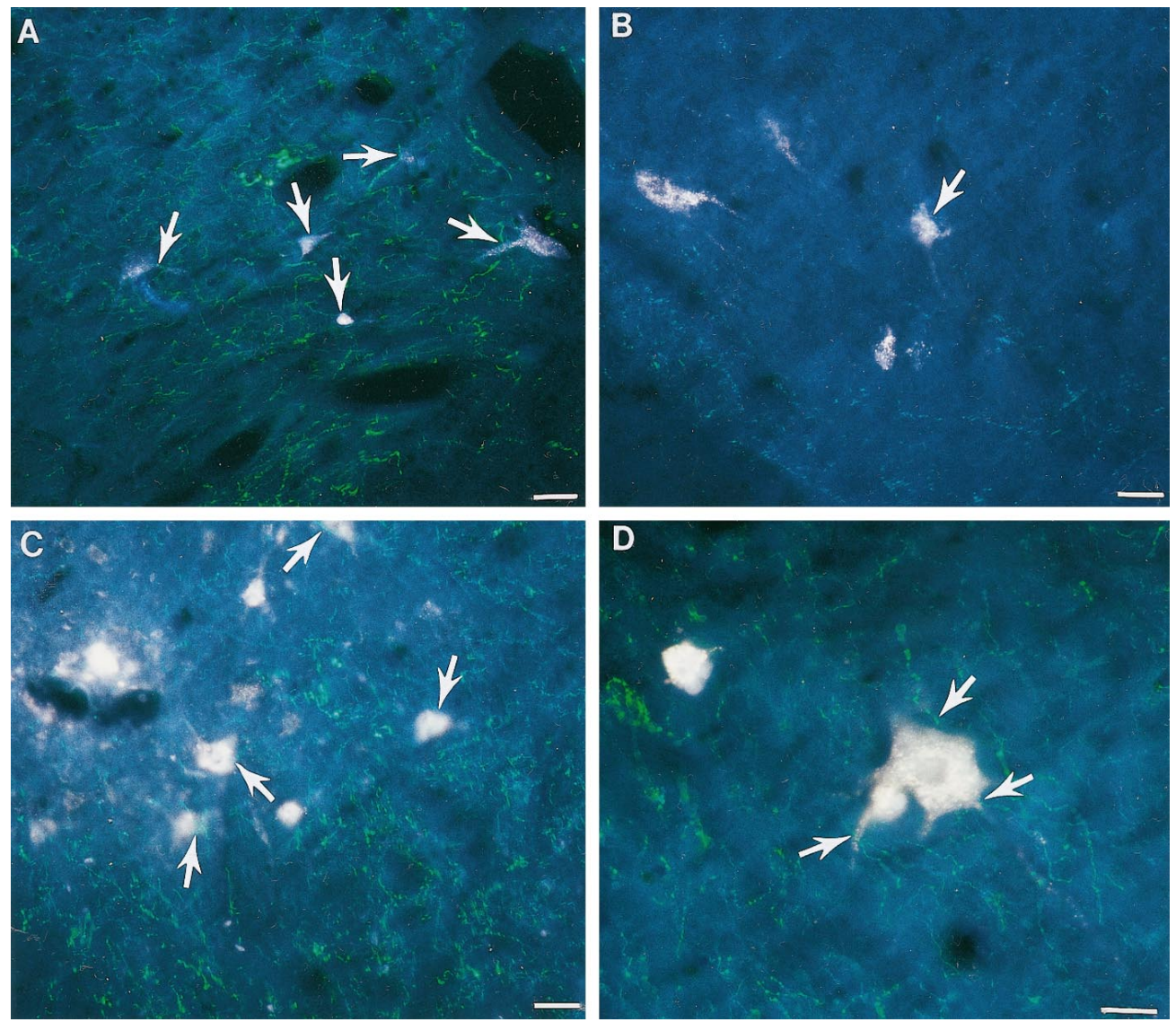

Figure 9. Effect of neonatal cervical spinal cord injury on the supraspinal projections to propriospinal neurons. Fluorescent photomicrographs of laminae VIII of double-labeled spinal cord sections from the $\mathrm{C} 5$ spinal cord segment. $A$, Representative section from a normal animal. Numerous propriospinal neurons (white cells) are labeled, and serotonergic axons (green fibers) are widely distributed throughout the ventral horn. Serotonergic axons are closely associated with the propriospinal neurons (arrows). $B$, In the HX group, although propriospinal neurons are retrogradely labeled (white cells), the serotonergic axons (green fibers) are substantially decreased in the ventral horn and are rarely associated with the propriospinal neurons (arrow depicts one cell with an adjacent axon). $C$, The $\mathrm{HX}+$ TP group has both labeled propriospinal neurons (white cells) and serotonergic axons (green fibers) distributed throughout the ventral horn. Many serotonergic axons are closely associated with the propriospinal neurons (arrows). D, Highpowered view of a representative propriospinal neuron (large white cell) with adjacent serotonergic fibers ( arrows) in a transplant animal. The presence of a transplant at the lesion site not only preserves more propriospinal neurons than in lesion-only animals, but the descending input to these neurons also seems to be reestablished. Scale bars, $50 \mu \mathrm{m}$. possibility that impaired forelimb use in lesion-only rats was caused by a reduction in the number of motoneurons was ruled out (see Materials and Methods and Results). More likely, poor abilities to reach and grasp and impaired postural control, as seen in the companion study (Diener and Bregman, 1998), are correlated with the loss of supraspinal input to spinal neurons, primarily brachial motoneurons, segmental interneurons, and short and long propriospinal neurons. The data in the current study as well as in other models support the idea that removal of supraspinal and segmental influence to spinal neurons results in impaired function (Alstermark et al., 1987a; Schrimsher and Reier, 1992, 1993). Axon growth back to target areas is associated with restoration of function (Bregman et al., 1991, 1993, 1995; for review, see Bregman, 1994; Howland et al., 1995).

The development of both reaching and its underlying neural network has not been examined previously in the neonatal rat. On the basis of current knowledge about the development of the propriospinal neurons in other species (Oppenheim et al., 1989; Cassidy and Cabana, 1993; Berkowitz and Stein, 1994), we hypothesized that the propriospinal neurons are present at birth in the rat. The studies reported here support this hypothesis. As in the chick (Oppenheim et al., 1989), opossum (Cassidy and Cabana, 1993), and turtle (Berkowitz and Stein, 1994), there is little postnatal reorganization of the laminar distribution of propriospinal neurons in the rat. The contribution of supraspinal projections to propriospinal neurons in the cervical cord was examined. We found that supraspinal projections are closely associated with numerous propriospinal neurons in rats in both the normal and transplant groups but not in lesion-only animals. We speculate that this close association between descending projections and spinal cord neurons develops or becomes functional postnatally. Postnatal development of supraspinal and segmental input to propriospinal neurons would be coincident with the postnatal acquisition of skilled forelimb use (Diener and Bregman, 1998) and therefore may provide a mechanism underlying target-directed reaching.

\section{Afferent contribution to the development of forelimb skills after neonatal injury and transplantation}

Supraspinal projections to propriospinal and other interneurons are not the only mechanism by which directed reaching and static or dynamic postural adjustments are guided. For example, primary afferent projections to the dorsal column nuclei appear to be important in producing the subtle muscle contractions necessary to make minor postural adjustments to maintain static postures (Reynolds et al., 1972). Limited neuroanatomical reorganization of ascending large diameter dorsal root projections (Lahr and Stelzner, 1990; our unpublished results) may have contributed to some of the aberrant behavior observed in the experimental animals [i.e., altered development of postural adjustments during the dynamic task of reaching (Diener and Bregman, 1998)]. Afferents ascending in the dorsal funiculus have terminated in the dorsal column nuclei by birth in the rodent (Lahr and Stelzner, 1990); consequently, these tracts are severed by neonatal cervical spinal cord injury. Severed lumbar dorsal root axons projecting to the gracilus nucleus do not regenerate after cervical spinal cord injury made within 30 hr after birth, although their cell bodies in the dorsal root ganglion survive (Lahr and Stelzner, 1990). In animals with lesions plus transplants, primary sensory axons retracted from the site of lesion or lesion plus transplant (Dent et al., 1996; our unpublished data). This lack of growth may explain both (1) the apparent impairment of proprioceptive feedback during reaching attempts observed occasionally in experimental rats on reaching attempts and (2) 


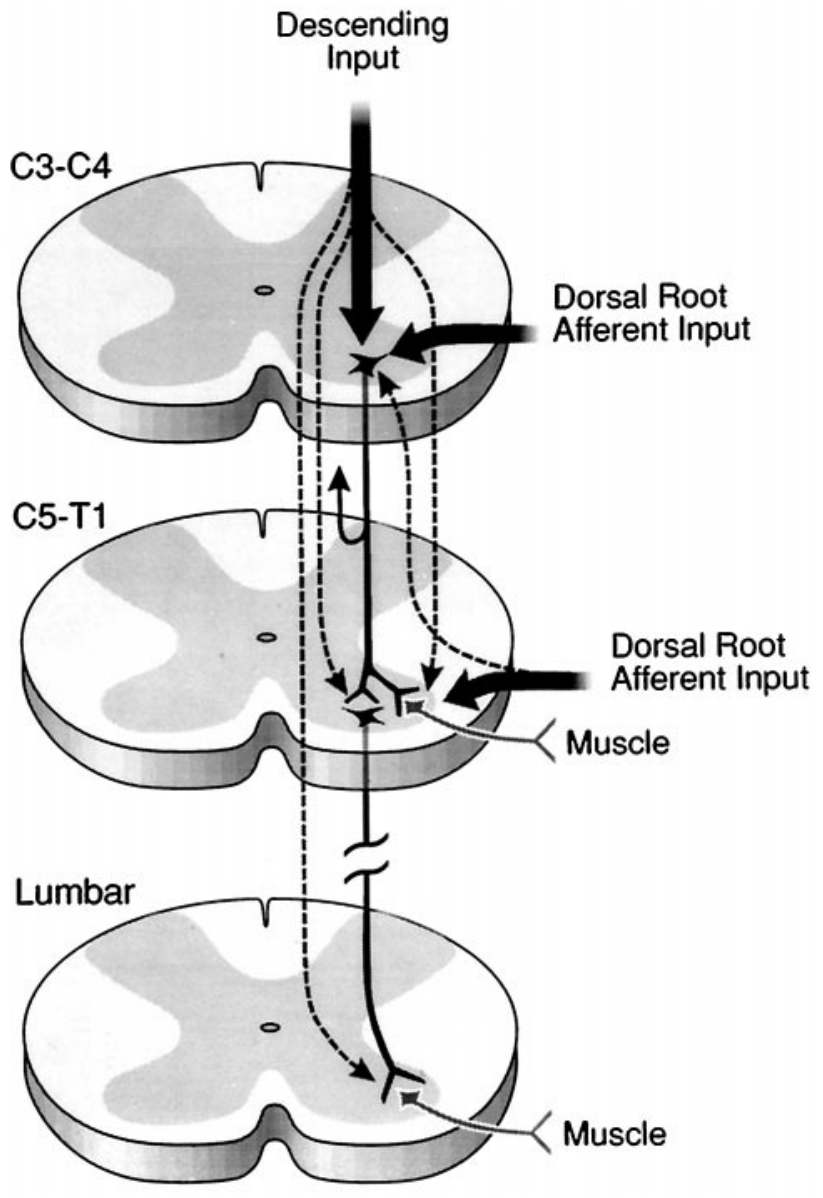

Figure 10. Summary diagram of a proposed mechanism that guides target reaching and postural adjustments after neonatal cervical spinal cord injury and transplantation. [Spinal cord levels are noted. Propriospinal neurons are represented as dark black neurons schematically located in the $\mathrm{C} 3-\mathrm{C} 4$ (short propriospinal neurons) and C5-T1 (long propriospinal neurons) segments. Supraspinal (descending) and segmental afferent input are labeled.] Before skilled movement emerges, neonatal high cervical spinal cord injury at $\mathrm{C} 3$ disrupts (1) the $\mathrm{C} 3-\mathrm{C} 4$ propriospinal network and (2) the supraspinal and segmental input directed to the $\mathrm{C} 3-\mathrm{C} 4$ propriospinal neurons, the forelimb and hindlimb segment motoneurons, and the interneurons. This schematic diagram represents some of the normal input that contributes to the propriospinal network, which we suggest is reestablished in the presence of a transplant growing within the C3 spinal cord segment. Because target reaching and postural adjustments develop postnatally, the proposed pathway is suggested also to develop after birth in the normal and transplant animals but not in lesion-only animals. The aberrant motor patterns exhibited by lesion-only rats (Diener and Bregman, 1998) are suggested to be commensurate with failed remodeling of the pathways identified in the schematic (refer to other photomicrographs in this paper) We suggest that through a transplant-mediated response, the descending and segmental projections to multiple spinal levels are reestablished (dashed arrows emanating from the descending and segmental arrows). The transplant may also mediate the extension of collaterals that normally would provide feedback to higher centers (indicated by the collateral with arrowhead curving upward from the $\mathrm{C} 3-\mathrm{C} 4$ propriospinal axon). After the converging input is integrated, the short $(\mathrm{C} 3-\mathrm{C} 4)$ propriospinal neurons may project to the brachial segments (possibly using the transplant as a bridge or a relay; refer to Figs. 3, 4, 5, 7, 8). All of these projections may influence forelimb motoneurons and long propriospinal neurons, which in turn may guide lower spinal cord segments. This schematic suggests a neural mechanism that may be reestablished after high spinal injury and transplantation to influence the development of postural adjustments (e.g., axial and hindlimb movements) in coordination with the development of forelimb movements (e.g., reaching), thereby furnishing an explanation for the skilled movement observed in the transplant rats (Diener and Bregman, 1998). aspects of disturbances in balancing the body (Diener and Bregman, 1998).

\section{Conclusion}

Novel findings from this and the companion study (Diener and Bregman, 1998) suggest that after neonatal cervical spinal cord injury, compensatory mechanisms develop that substitute for failed acquisition of skilled movement and anticipatory and dynamic postural adjustments. We suggest that these behavioral impairments are attributable to diminished axonal growth after injury and reduced input to spinal cord circuitry underlying skilled forelimb use. In contrast, the acquisition of target-guided movements and anticipatory and dynamic postural adjustments demonstrated by the transplant animals suggest that the long-distance growth and the close approximation of axons to appropriate anatomical targets underlie the developed movements. Moreover, the modest reduction in the density of remodeled fibers in target areas may be one explanation for the qualitative differences seen in skilled movement patterns in transplant and normal animals.

\section{REFERENCES}

Alstermark B, Sasaki S (1985) Integration in descending motor pathways controlling the forelimb in the cat. 13. Corticospinal effects in shoulder: elbow, wrist, and digit motoneurones. Exp Brain Res 59:353-364.

Alstermark B, Wessberg J (1985) Timing of postural adjustment in relation to forelimb target-reaching in cats. Acta Physiol Scand 125:337-340.

Alstermark B, Lindstrom S, Lundberg A, Sybirska E (1981a) Integration in descending motor pathways controlling the forelimb in the cat. 8 . Ascending projection to the lateral reticular nucleus from $\mathrm{C} 3-\mathrm{C} 4$ propriospinal neurones also projecting to forelimb motoneurones. Exp Brain Res 42:282-298.

Alstermark B, Lundberg A, Norrsell U, Sybirska E (1981b) Integration in descending motor pathways controlling the forelimb in the cat. 9 . Differential behavioural defects after spinal cord lesions interrupting defined pathways from higher centres to motoneurones. Exp Brain Res 42:299-318.

Alstermark B, Lundberg A, Sasaki S (1984a) Integration in descending motor pathways controlling the forelimb in the cat. 11. Inhibitory pathways from higher motor centres and forelimb afferents to $\mathrm{C} 3-\mathrm{C} 4$ propriospinal neurones. Exp Brain Res 56:293-307.

Alstermark B, Lundberg A, Sasaki S (1984b) Integration in descending motor pathways controlling forelimb in the cat. 12. Interneurones which may mediate descending feed-forward inhibition and feed-back inhibition from the forelimb to $\mathrm{C} 3-\mathrm{C} 4$ propriospinal neurones. Exp Brain Res 56:308-322.

Alstermark B, Lundberg A, Pettersson L-G, Tantisira B, Walkowska M (1987a) Motor recovery after serial spinal cord lesions of defined descending pathways in cats. Neurosci Res 5:68-73.

Alstermark B, Lundberg A, Pinter M, Sasaki S (1987b) Long C3-C5 propriospinal neurones in the cat. Brain Res 404:382-388.

Alstermark B, Lundberg A, Pinter M, Sasaki S (1987c) Vestibular effects in long C3-C5 propriospinal neurones. Brain Res 404:389-394.

Alstermark B, Lundberg A, Pinter M, Sasaki S (1987d) Subpopulations and functions of long $\mathrm{C} 3-\mathrm{C} 5$ propriospinal neurones. Brain Res 404:395-400.

Alstermark B, Kummel H, Pinter MJ, Tantisira B (1990) Integration in descending motor pathways controlling the forelimb in the cat. 17. Axonal projection and termination of $\mathrm{C} 3-\mathrm{C} 4$ propriospinal neurons in the C6-T1 segments. Exp Brain Res 81: 447-461.

Alstermark B, Isa T, Tantisira B (1991a) Pyramidal excitation in long propriospinal neurones in the cervical segments of the cat. Exp Brain Res 84:569-582.

Alstermark B, Isa T, Tantisira B (1991b) Integration in descending motor pathways controlling the forelimb in the cat. 18. Morphology: axonal projection and termination of collaterals from C3-C4 propriospinal neurones in the segment of origin. Exp Brain Res 84: 561-568.

Andersson O, Grillner S, Lindquist M, Zomlefer M (1978) Peripheral control of the spinal pattern generators for locomotion in cat. Brain Res 150:625-630.

Berkowitz A, Stein PSG (1994) Descending propriospinal axons in the 
hindlimb enlargement of the red-eared turtle: cells of origin and funicular courses. J Comp Neurol 346:321-336.

Bernhard CG, Rexed B (1945) The localization of the premotor interneurons discharging through the peroneal nerve. J Neurophysiol 8:387-392.

Bernstein-Goral H, Bregman BS (1993) Spinal cord transplants support the regeneration of axotomized neurons after spinal cord lesions at birth: a quantitative double-labeling study. Exp Neurol 123:118-132.

Bernstein-Goral H, Bregman BS (1997) Axotomized rubrospinal neurons rescued by fetal spinal cord transplants maintain axon collaterals to rostral CNS targets. Exp Neurol 148:13-25.

Bernstein-Goral H, Diener PS, Bregman BS (1997) Regenerating and sprouting axons differ in their requirements for growth after injury. Exp Neurol 148:51-72.

Bregman BS (1987a) Development of serotonin immunoreactivity in the rat spinal cord and its plasticity after neonatal spinal cord lesions. Brain Res 431:245-263.

Bregman BS (1987b) Spinal cord transplants permit the growth or serotonergic axons across the site of neonatal spinal cord transection. Dev Brain Res 34:265-279.

Bregman BS (1994) Recovery of function after spinal cord injury: transplantation strategies. In: Functional neural transplantation (Dunnett SB, Bjorkland A, eds), pp 489-529. New York: Raven.

Bregman BS, Bernstein-Goral H (1991) Both regenerating and latedeveloping pathways contribute to transplant-induced anatomical plasticity after spinal cord lesions at birth. Exp Neurol 112:49-63.

Bregman BS, Goldberger ME (1983) Infant lesion effect: III. Anatomical correlates of sparing and recovery of function after spinal cord damage in newborn and adult cats. Dev Brain Res 9:137-154.

Bregman BS, McAtee M (1993) Embryonic CNS tissue transplantation for studies of development and regeneration. Neuroprotocols 3:17-27.

Bregman BS, Reier PJ (1986) Neural tissue transplants rescue axotomized rubrospinal cells from retrograde death. J Comp Neurol 244:86-95.

Bregman BS, Kunkel-Bagden E, McAtee M, O’Neill A (1989) Extension of the critical period for developmental plasticity of the corticospinal pathway. J Comp Neurol 282:355-370.

Bregman BS, Bernstein-Goral H, Kunkel-Bagden E (1991) CNS transplants promote anatomical plasticity and recovery of function after spinal cord injury. Restor Neurol Neurosci 2:327-338.

Bregman BS, Kunkel-Bagden E, Reier PJ, Dai HN, McAtee M, Gao D (1993) Recovery of function after spinal cord injury: mechanisms underlying transplant-mediated recovery of function differ after spinal cord injury in newborn and adult rats. Exp Neurol 123:3-16.

Bregman BS, Kunkel-Bagden E, Schnell L, Dai HN, Gao D, Schwab ME (1995) Recovery from spinal cord injury mediated by antibodies to neurite growth inhibitors. Nature 378:498-501.

Bregman BS, Diener PS, McAtee M, Dai HN, James C (1997) Intervention strategies to enhance anatomical plasticity and recovery of function after spinal cord injury. Adv in Neurol 72:257-275.

Cassidy G, Cabana T (1993) The development of the long descending propriospinal projections in the opossum, Monodelphis Domestica. Dev Brain Res 72:291-299.

Castro AJ (1972a) The effects of cortical ablations on digital usage in the rat. Brain Res 37:173-185.

Castro AJ (1972b) Motor performance in rats: the effects of pyramidal tract section. Brain Res 44:313-323.

Dent LJB, McCasland JS, Stelzner DJ (1996) Attempts to facilitate dorsal column axonal regeneration in a neonatal spinal environment. J Comp Neurol 372:435-456.

Diener PS, Bregman BS (1994) Neurotrophic factors prevent the death of CNS neurons after spinal cord lesions in newborn rats. NeuroReport 5:1913-1917.

Diener PS, Bregman BS (1995) Fetal spinal cord transplants mediate anatomical reorganization and support the development of target reaching and coordinated postural adjustments after cervical spinal cord injury in neonatal rats. Soc Neurosci Abstr 21:1309.

Diener PS, Bregman BS (1998) Fetal spinal cord transplants support the development of target reaching and coordinated postural adjustments after neonatal cervical spinal cord injury. J Neurosci 18:763-778.

Evans BH, Ingram WR (1939) The effects of combined red nucleus and pyramidal lesions in cats. J Comp Neurol 70:461-476.

Ghez C, Martin JH (1982) The control of rapid limb movement in the cat. III. Agonist-antagonist coupling. Exp Brain Res 45:115-125.

Goldberger ME, Murray M (1985) Recovery of functional and anatomical plasticity after damage to the adult and neonatal spinal cord. In: Synaptic plasticity (Cotman CW, ed), pp 77-110. New York: Guilford.
Grillner S (1973) Locomotion in the spinal cat. In: Control of posture and locomotion (Stein RB, Pearson KG, Smith RS, Redford JB, eds), pp 515-535. New York: Plenum.

Grillner S (1975) Locomotion in vertebrates: central mechanisms and reflex interaction. Physiol Rev 55:247-304.

Howland DR, Bregman BS, Tessler A, Goldberger ME (1995) Transplants enhance locomotion in neonatal kittens whose spinal cords are transected: a behavioral and anatomical study. Exp Neurol 135:123-145.

Illert M, Tanaka R (1978) Integration in descending motor pathways controlling the forelimb in the cat. 4. Corticospinal inhibition of forelimb motoneurones mediated by short propriospinal neurones. Exp Brain Res 31:131-141.

Illert M, Lundberg A, Tanaka R (1976a) Integration in descending motor pathways controlling the forelimb in the cat. 2. Convergence on neurones mediating disynaptic cortico-motoneuronal excitation. Exp Brain Res 26:521-540.

Illert M, Lundberg A, Tanaka R (1976b) Integration in descending motor pathways controlling the forelimb in the cat. 1. Pyramidal effects on motoneurones. Exp Brain Res 26:509-519.

Itoh Y, Tessler A (1990) Regeneration of adult dorsal root axons into transplants of fetal spinal cord and brain: a comparison of growth and synapse formation in appropriate and inappropriate targets. J Comp Neurol 302:272-293.

Jakeman LB, Reier PJ (1991) Axonal projections between fetal spinal cord transplants and the adult rat spinal cord: a neuroanatomical tracing study of local interactions. J Comp Neurol 307:311-334.

Jakeman LB, Reier PJ, Bregman BS, Wade EB, Kastner RJ, Dailey M (1989) Differentiation of substantia gelatinosa-like regions in intraspinal and intracerebral transplants of embryonic spinal cord tissue in the rat. Exp Neurol 103:17-33.

Kunkel-Bagden E, Bregman BS (1990) Spinal cord transplants enhance the recovery of locomotor function after spinal cord injury at birth. Exp Brain Res 81:25-34.

Kunkel-Bagden E, Dai HN, Bregman BS (1992) Recovery of function after spinal cord hemisection in newborn and adult rats: differential effects on reflex and locomotor function. Exp Neurol 116:40-51.

Lahr SP, Stelzner DJ (1990) Anatomical studies of dorsal column axons and dorsal root ganglion cells after spinal cord injury in the newborn rat. J Comp Neurol 293:377-398.

Lawrence DG, Kuypers HGJM (1968a) The functional organization of the motor system in the monkey. I. The effects of bilateral pyramidal lesions. Brain 91:1-14.

Lawrence DG, Kuypers HGJM (1968b) The functional organization of the motor system in the monkey. II. The effects of lesions of the descending brain stem pathways. Brain 91:15-36.

Martin JH (1993) Differential spinal projections of subregions in the forelimb areas of motor cortex in the cat. Neurosci Lett 159:195-198.

Martin JH, Ghez C (1993) Differential impairments in reaching and grasping produced by local inactivation within the forelimb representation of the motor cortex in the cat. Exp Brain Res 94:429-443.

Oppenheim RW, Shneiderman A, Shimizu I, Yaginuma H (1989) Onset and development of intersegmental projections in the chick embryo spinal cord. J Comp Neurol 275:159-180.

Reier PJ, Bregman BS, Wujek JR (1986) Intraspinal transplantation of embryonic spinal cord tissue in neonatal and adult rats. J Comp Neurol 247:275-296.

Reynolds PJ, Talbott RE, Brookhart JM (1972) Control of postural reactions in the dog: the role of the dorsal column feedback pathway. Brain Res 40:159-164.

Schrimsher GW, Reier PJ (1992) Forelimb motor performance following cervical spinal cord contusion injury in the rat. Exp Neurol 117:287-298.

Schrimsher GW, Reier PJ (1993) Forelimb motor performance following dorsal column, dorsolateral funiculi, or ventrolateral funiculi lesions of the cervical spinal cord in the rat. Exp Neurol 120:264-276.

Schwab ME, Bartholdi D (1996) Degeneration and regeneration of axons in the lesioned spinal cord. Physiol Rev 76:319-370.

Schwab ME, Kapfhammer JP, Bandtlow CE (1993) Inhibitors of neurite growth. Annu Rev Neurosci 16:565-595.

Sterling P, Kuypers HGJM (1968) Anatomical organization of the brachial spinal cord of the cat. III. The propriospinal connections. Brain Res 7:419-443.

Sternberger LA (1976) Immunocytochemistry. New York: Wiley.

Waldron HA (1969) Descending nerve tracts in the spinal cord of the rat. I. Fibers from the midbrain. J Comp Neurol 137:143-154. 